\title{
Stabilized CutFEM for the convection problem on surfaces
}

\author{
Erik Burman ${ }^{1} \cdot$ Peter Hansbo ${ }^{2}$ - Mats G. Larson ${ }^{3} \cdot$ Sara Zahedi $^{4}$ \\ Received: 7 November 2015 / Revised: 24 February 2017 / Published online: 18 August 2018 \\ (c) The Author(s) 2018
}

\begin{abstract}
We develop a stabilized cut finite element method for the convection problem on a surface based on continuous piecewise linear approximation and gradient jump stabilization terms. The discrete piecewise linear surface cuts through a background mesh consisting of tetrahedra in an arbitrary way and the finite element space consists of piecewise linear continuous functions defined on the background mesh. The variational form involves integrals on the surface and the gradient jump stabilization term is defined on the full faces of the tetrahedra. The stabilization term serves two purposes: first the method is stabilized and secondly the resulting linear system of equations is algebraically stable. We establish stability results that are analogous to the standard meshed flat case and prove $h^{3 / 2}$ order convergence in the natural norm associated with the method and that the full gradient enjoys $h^{3 / 4}$ order of convergence in $L^{2}$. We also show that the condition number of the stiffness matrix is bounded by $h^{-2}$. Finally, our results are verified by numerical examples.
\end{abstract}

Mathematics Subject Classification $65 \mathrm{~N} 30 \cdot 65 \mathrm{~N} 85$

\section{Contents}

1 Introduction . . . . . . . . . . . . . . . . . . . . . . . 104

2 The convection problem on a surface . . . . . . . . . . . . . . . 105

This research was supported in part by the Swedish Foundation for Strategic Research Grant No. AM13-0029 (PH,MGL), the Swedish Research Council Grants Nos. 2011-4992 (PH), 2013-4708 (MGL), and 2014-4804 (SZ), the Swedish Research Programme Essence (MGL, SZ), and EPSRC, UK, Grant Nr. $\mathrm{EP} / \mathrm{J} 002313 / 2$. (EB).

$凶$ Mats G. Larson

mats.larson@math.umu.se

1 Department of Mathematics, University College London, London WC1E 6BT, UK

2 Department of Mechanical Engineering, Jönköping University, 55111 Jönköping, Sweden

3 Department of Mathematics and Mathematical Statistics, Umeå University, 90187 Umeå, Sweden

4 Department of Mathematics, KTH Royal Institute of Technology, 10044 Stockholm, Sweden 


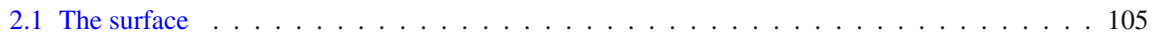

2.2 Tangential calculus . . . . . . . . . . . . . . . . . . . . 106

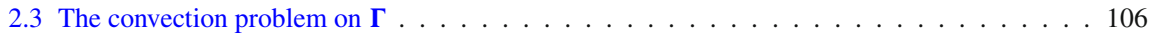

3 The finite element method . . . . . . . . . . . . . . . . . . . 108

3.1 The discrete surface . . . . . . . . . . . . . . . . . . . . . . . . . 108

3.2 The finite element method . . . . . . . . . . . . . . . . . . . . . . . 109

4 Preliminary results . . . . . . . . . . . . . . . . . . . . . . . 110

4.1 Norms . . . . . . . . . . . . . . . . . . . . . . 110

4.2 Inverse estimates . . . . . . . . . . . . . . . . . . . . 110

4.3 Extension and lifting of functions . . . . . . . . . . . . . . . . . 111

4.4 Interpolation . . . . . . . . . . . . . . . . . . . . . . . 112

5 Stability estimates . . . . . . . . . . . . . . . . . . . . 113

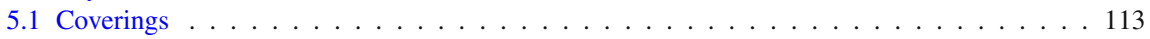

5.2 Assumptions on the coefficients for the stability estimates . . . . . . . . . . . 116

5.3 Technical lemmas . . . . . . . . . . . . . . . . . . . . . . . . . . . . . . . . . . . . . . . . . . . . . . . . .

5.4 Stability estimates . . . . . . . . . . . . . . . . . . . . . . . . 119

6 Error estimates . . . . . . . . . . . . . . . . . . . . . . . . 125

6.1 Assumptions on the coefficients for the error estimates . . . . . . . . . . . 125

6.2 Strang's Lemma . . . . . . . . . . . . . . . . . . . . . . . . . . . . . . . . . . . . . . . . . . . . . . . . . . . . . . . . . . . . . . . . .

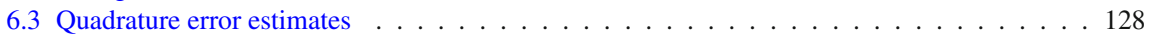

6.4 Construction of the discrete coefficients . . . . . . . . . . . . . . . . . . . . 129

6.5 Error estimates . . . . . . . . . . . . . . . . . . . . . . . 132

7 Condition number estimate . . . . . . . . . . . . . . . . . . 133

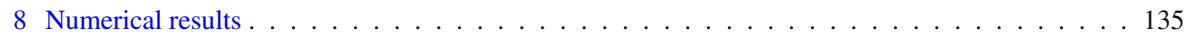

8.1 Convergence study . . . . . . . . . . . . . . . . . . . . . . . 138

8.2 Condition number study . . . . . . . . . . . . . . . . . . . . 138

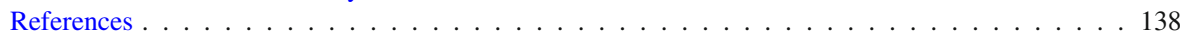

\section{Introduction}

In this contribution we develop a stabilized cut finite element for stationary convection on a surface embedded in $\mathbb{R}^{3}$. The method is based on a three dimensional background mesh consisting of tetrahedra and a piecewise linear approximation of the surface. The finite element space is the continuous piecewise linear functions on the background mesh and the bilinear form defining the method only involves integrals on the surface. In addition we add a consistent stabilization term which involves the normal gradient jump on the full faces of the background mesh. In the case of the Laplace-Beltrami operator the idea of using the restriction of a finite element space to the surface was developed in [23], and a stabilized version was proposed and analyzed in [6].

We show that for the convection problem the properties of cut finite element method completely reflects the properties of the corresponding method on standard triangles or tetrahedra, see the analysis for the latter in [3]. In particular, we prove discrete stability estimates in the natural energy norm, involving the $L^{2}$ norm of the solution and $h^{1 / 2}$ times the $L^{2}$ norm of the streamline derivative where $h$ is the meshsize, and corresponding optimal a priori error estimates of order $h^{3 / 2}$. Furthermore, we also show an error estimate of order $h^{3 / 4}$ for the error in the full gradient which is also in line with [3]. The stabilization term is key to the proof of the discrete stability estimates and enables us to work in the natural norms corresponding to those used in the standard analysis on triangles or tetrahedra. The analysis utilizes a covering argument first developed in [6], which essentially localizes the analysis to sets of 
elements, with a uniformly bounded number of elements, that together has properties similar to standard finite elements. The stabilization term also leads to an algebraically stable linear system of equations and we prove that the condition number is bounded by $h^{-2}$.

We note that similar stabilization terms have recently been used for stabilization of cut finite element methods for time dependent problems in [20], bulk domain problems involving standard boundary and interface conditions [4,5,18,21], and for coupled bulk-surface problems involving the Laplace-Beltrami operator on the surface in [8]. We also mention [7] where a discontinuous cut finite element method for the Laplace-Beltrami operator was developed. None of these references consider the convection problem on the surface. For convection problems streamline diffusion stabilization was used in [9,25]. Methods on evolving surfaces were studied in $[20,22,24]$.

An advantage of the proposed stabilization method is that it is straightforward to extend the method to a time dependent problem on a stationary surface. Indeed any A-stable finite difference discretization of the time derivative leads to a stable scheme with the accuracy of the truncation error [2]. Runge-Kutta methods of second and third orders are also stable and accurate and also explicit up to the inversion of the mass matrix [1]. In the explicit case the mass matrix is stabilized using a scaled version of the normal gradient jump term. For time-dependent domains on the other hand it may be more convenient to use the aforementioned space-time finite elements for a consistent tracking of the surface displacement [20,22] or a combination with the characteristic approach developed in [19].

Finally, we refer to [10,13-15] for general background on finite element methods for partial differential equations on surfaces.

The outline of the remainder of this paper is as follows: In Sect. 2 we formulate the model problem; in Sect. 3 we define the discrete surface, its approximation properties, and the finite element method; in Sect. 4 we summarize some preliminary results involving lifting of functions from the discrete surface to the continuous surface; in Sect. 5 we first derive some technical lemmas essentially quantifying the stability induced by the stabilization term, and then we derive the key discrete stability estimate; in Sect. 6 we prove a priori estimates; in Sect. 7 we prove an estimate of the condition number; and finally in Sect. 8, we present some numerical examples illustrating the theoretical results.

\section{The convection problem on a surface}

\subsection{The surface}

Let $\Gamma$ be a smooth surface embedded in $\mathbb{R}^{3}$ with signed distance function $\rho$ such that the exterior unit normal to the surface is given by $n=\nabla \rho$. We let $p: \mathbb{R}^{3} \rightarrow \Gamma$ be the closest point mapping. Then there is a $\delta_{0}>0$ such that $p$ maps each point in $U_{\delta_{0}}(\Gamma)$ to precisely one point on $\Gamma$, where $U_{\delta}(\Gamma)=\left\{x \in \mathbb{R}^{3}:|\rho(x)|<\delta\right\}$ is the open tubular neighborhood of $\Gamma$ of thickness $\delta$. 


\subsection{Tangential calculus}

For each function $u$ on $\Gamma$ we let the extension $u^{e}$ to the neighborhood $U_{\delta_{0}}(\Gamma)$ be defined by the pull back $u^{e}=u \circ p$. For a function $u: \Gamma \rightarrow \mathbb{R}$ we then define the tangential gradient

$$
\nabla_{\Gamma} u=P_{\Gamma} \nabla u^{e}
$$

where $P_{\Gamma}=I-n \otimes n$, with $n=n(x), x \in \Gamma$, in the projection onto the tangent plane $T_{x}(\Gamma)$. We also define the surface divergence

$$
\operatorname{div}_{\Gamma}(u)=\operatorname{tr}\left(u \otimes \nabla_{\Gamma}\right)=\operatorname{tr}\left(u^{e} \otimes \nabla P_{\Gamma}\right)
$$

where $\left(u^{e} \otimes \nabla\right)_{i j}=\partial_{j} u_{i}^{e}$. It can be shown that the tangential derivative does not depend on the particular choice of extension.

\subsection{The convection problem on $\Gamma$}

The strong form of the convection problem on $\Gamma$ takes the form: find $u: \Gamma \rightarrow \mathbb{R}$ such that

$$
\beta \cdot \nabla_{\Gamma} u+\alpha u=f \quad \text { on } \Gamma
$$

where $\beta: \Gamma \rightarrow \mathbb{R}^{3}$ is a given tangential vector field, $\alpha: \Gamma \rightarrow \mathbb{R}$ and $f: \Gamma \rightarrow \mathbb{R}$ are given functions.

Assumption The coefficients $\alpha \in C(\Gamma)$ and $\beta \in C^{1}(\Gamma)$ satisfy

$$
0<C \leq \inf _{x \in \Gamma}\left(\alpha(x)-\frac{1}{2} \operatorname{div}_{\Gamma} \beta(x)\right)
$$

for a positive constant $C$.

We introduce the Hilbert space $V=\left\{v: \Gamma \rightarrow \mathbb{R}:\|v\|_{V}^{2}=\|v\|_{\Gamma}^{2}+\|\beta \cdot \nabla v\|_{\Gamma}^{2}<\infty\right\}$ and the operator $L: V \ni v \mapsto \beta \cdot \nabla_{\Gamma} v+\alpha v \in L^{2}(\Gamma)$. We note that using Green's formula and assumption (2.4) we have the estimate

$$
(L v, v)_{\Gamma}=\left(\left(\alpha-\frac{1}{2} \operatorname{div}_{\Gamma} \beta\right) v, v\right)_{\Gamma} \geq C\|v\|_{\Gamma}^{2}
$$

Proposition 2.1 If the coefficients $\alpha$ and $\beta$ satisfy assumption (2.4), then there is a unique $u \in V$ such that $L u=f$ for each $f \in L^{2}(\Gamma)$.

Proof The essential idea in the proof is to consider the corresponding time dependent problem with a smooth right hand side and show that the solution exists and converges to a solution to the stationary problem as time tends to infinity. Then we use a density argument to handle a right hand side in $L^{2}$. 
Smooth right hand side For any $0<T<\infty$ consider the time dependent problem: find $u:[0, T] \times \Gamma \rightarrow \mathbb{R}$, such that

$$
u_{t}+\beta \cdot \nabla_{\Gamma} u+\alpha u=g \quad \text { on }(0, T] \times \Gamma, \quad u(0)=0 \quad \text { on } \Gamma
$$

Consider first a smooth right hand side $g$, which does not depend on time. Using characteristic coordinates we conclude that there is smooth solution $u(t)$ to (2.6). Next taking the time derivative of Eq. (2.6) we find that the solution satisfies the equation

$$
u_{t t}+\beta \cdot \nabla_{\Gamma} u_{t}+\alpha u_{t}=0
$$

where we used the fact that $\alpha, \beta$, and $g$, do not depend on time. Multiplying (2.7) by $u_{t}$ and integrating over $\Gamma$ we get

$$
\frac{d}{d t}\left\|u_{t}\right\|_{\Gamma}^{2}+\left(\left(2 \alpha-\operatorname{div}_{\Gamma} \beta\right) u_{t}, u_{t}\right)_{\Gamma}=0
$$

Using (2.4) we obtain

$$
\frac{d}{d t}\left\|u_{t}\right\|_{\Gamma}^{2}+2 C\left\|u_{t}\right\|_{\Gamma}^{2} \leq 0
$$

which implies

$$
\frac{d}{d t}\left(\left\|u_{t}\right\|_{\Gamma}^{2} e^{2 C t}\right) \leq 0
$$

Integrating over $[\epsilon, T], 0<\epsilon<T$, we get

$$
\left\|u_{t}(T)\right\|_{\Gamma} \leq\left\|u_{t}(\epsilon)\right\|_{\Gamma} e^{-2 C(T-\epsilon)}
$$

Letting $\epsilon \rightarrow 0^{+}$and using the smoothness of $u$ we find, using the Eq. (2.6), that $u_{t}(\epsilon)=g-\beta \cdot \nabla_{\Gamma} u(\epsilon)-\alpha u(\epsilon) \rightarrow g-\beta \cdot \nabla_{\Gamma} u(0)-\alpha u(0)=g$ since $u(0)=0$ and therefore also $\nabla_{\Gamma} u(0)=0$. We thus conclude that

$$
\left\|u_{t}(T)\right\|_{\Gamma} \leq\|g\|_{\Gamma} e^{-2 C T}
$$

Using (2.12) we have

$$
\begin{aligned}
& \left\|u\left(T_{2}\right)-u\left(T_{1}\right)\right\|_{\Gamma}=\left\|\int_{T_{1}}^{T_{2}} u_{t}(s) d s\right\|_{\Gamma} \leq \int_{T_{1}}^{T_{2}}\left\|u_{t}(s)\right\|_{\Gamma} d s \\
& \leq \int_{T_{1}}^{T_{2}}\|g\|_{\Gamma} e^{-2 C s} d s \leq(2 C)^{-1} e^{-2 C T_{1}}\left(1+e^{-2 C\left(T_{2}-T 1\right)}\right)\|g\|_{\Gamma} \lesssim e^{-2 C T_{1}}\|g\|_{\Gamma}
\end{aligned}
$$

for $0 \leq T_{1} \leq T_{2}<\infty$. Using the time dependent Eq. (2.6) we have

$$
\beta \cdot \nabla\left(u\left(T_{2}\right)-u\left(T_{1}\right)\right)=u_{t}\left(T_{1}\right)-u_{t}\left(T_{2}\right)+\alpha\left(u\left(T_{1}\right)-u\left(T_{2}\right)\right)
$$


and therefore, using the fact that $\|\alpha\|_{L^{\infty}(\Gamma)} \lesssim 1$, we have the estimate

$$
\begin{aligned}
\left\|\beta \cdot \nabla\left(u\left(T_{2}\right)-u\left(T_{1}\right)\right)\right\|_{\Gamma} & \lesssim\left\|u_{t}\left(T_{1}\right)\right\|_{\Gamma}+\left\|u_{t}\left(T_{2}\right)\right\|_{\Gamma}+\left\|u\left(T_{2}\right)-u\left(T_{1}\right)\right\|_{\Gamma} \\
& \lesssim e^{-2 C T_{1}}\|g\|_{\Gamma}
\end{aligned}
$$

where we used (2.12) and (2.14) in the last step. Together, (2.14) and (2.17) leads to the estimate

$$
\left\|u\left(T_{2}\right)-u\left(T_{1}\right)\right\|_{V} \lesssim e^{-2 C T_{1}}\|g\|_{\Gamma}, \quad 0 \leq T_{1} \leq T_{2}
$$

Thus we conclude that for each $\epsilon>0$ there is $T_{\epsilon}$ such that $\left\|u\left(T_{1}\right)-u\left(T_{2}\right)\right\|_{V} \leq \epsilon$ for all $T_{1}, T_{2}>T_{\epsilon}$. We can then pick a sequence $u_{n}=u\left(T_{n}\right)$ with $T_{n}=n, n=1,2,3, \ldots$ and conclude from (2.18) that the sequence is Cauchy in $V$ and therefore it converges to a limit $u_{g} \in V$. We then have

$$
\begin{aligned}
\left\|L u_{g}-g\right\|_{\Gamma} & \leq\left\|L u_{g}-L u_{n}\right\|_{\Gamma}+\left\|L u_{n}-g\right\|_{\Gamma} \\
& \leq\left\|u_{g}-u_{n}\right\|_{V}+\left\|u_{t}\left(T_{n}\right)\right\|_{\Gamma} \leq e^{-2 C T_{n}}\|g\|_{\Gamma}
\end{aligned}
$$

and thus the limit $u_{g}$ is a solution to the stationary problem in the sense of $L^{2}$ and from (2.18) with $T_{1}=0$, we have the stability estimate

$$
\left\|u_{g}\right\|_{V} \lesssim\|g\|_{\Gamma}
$$

Right hand side in $L^{\mathbf{2}}(\Gamma)$ For $f \in L^{2}(\Gamma)$ we pick a sequence of smooth functions $f_{n}$ that converges to $f$ in $L^{2}(\Gamma)$. Then for each $f_{n}$ there is a solution $u_{n} \in V$ to $L u_{n}=f_{n}$ and we note that $L\left(u_{n}-u_{m}\right)=f_{n}-f_{m}$ and therefore it follows from (2.20) that

$$
\left\|u_{n}-u_{m}\right\|_{V} \lesssim\left\|f_{n}-f_{m}\right\|_{\Gamma}
$$

and thus $\left\{u_{n}\right\}$ is a Cauchy sequence since $\left\{f_{n}\right\}$ is a Cauchy sequence. Denoting the limit of $u_{n}$ by $u$ we have

$$
\|L u-f\|_{\Gamma} \leq\left\|L\left(u-u_{n}\right)\right\|_{\Gamma}+\left\|f_{n}-f\right\|_{\Gamma} \leq\left\|u-u_{n}\right\|_{V}+\left\|f_{n}-f\right\|_{\Gamma}
$$

which tends to zero as $n$ tends to infinity and thus $u \in V$ is a solution to $L u=f$ in the sense of $L^{2}$.

\section{The finite element method}

\subsection{The discrete surface}

Let $\Omega_{0}$ be a polygonal domain that contains $U_{\delta_{0}}(\Gamma)$ and let $\left\{\mathcal{T}_{0, h}, h \in\left(0, h_{0}\right]\right\}$ be a family of quasiuniform partitions of $\Omega_{0}$ into shape regular tetrahedra with mesh parameter $h$. Let $\Gamma_{h} \subset \Omega_{0}$ be a connected surface such that $\Gamma_{h} \cap T$ is a subset of some hyperplane for each $T \in \mathcal{T}_{0, h}$ and let $n_{h}$ be the piecewise constant unit normal to $\Gamma_{h}$. 
Geometric approximation property The family $\left\{\Gamma_{h}: h \in\left(0, h_{0}\right]\right\}$ approximates $\Gamma$ in the following sense:

- $\Gamma_{h} \subset U_{\delta_{0}}(\Gamma), \forall h \in\left(0, h_{0}\right]$, and the closest point mapping $p: \Gamma_{h} \rightarrow \Gamma$ is a bijection.

- The following estimates hold

$$
\|\rho\|_{L^{\infty}\left(\Gamma_{h}\right)} \lesssim h^{2}, \quad\left\|n-n_{h}\right\|_{L^{\infty}\left(\Gamma_{h}\right)} \lesssim h
$$

We introduce the following notation for the geometric entities involved in the mesh

$$
\begin{aligned}
\mathcal{T}_{h} & =\left\{T \in \mathcal{T}_{h, 0}: \bar{T} \cap \Gamma_{h} \neq \emptyset\right\} \\
\mathcal{F}_{h} & =\left\{F=\left(\bar{T}_{1} \cap \bar{T}_{2}\right) \backslash \partial\left(\bar{T}_{1} \cap \bar{T}_{2}\right): T_{1}, T_{2} \in \mathcal{T}_{h}\right\} \\
\mathcal{K}_{h} & =\left\{K=T \cap \Gamma_{h}: T \in \mathcal{T}_{h}\right\} \cup\left\{F \in \mathcal{F}_{h}: F \subset \Gamma_{h}\right\} \\
\mathcal{E}_{h} & =\left\{E=\partial K_{1} \cap \partial K_{2}: K_{1}, K_{2} \in \mathcal{K}_{h}\right\}
\end{aligned}
$$

We also use the notation $\omega^{l}=\left\{p(x) \in \Gamma: x \in \omega \subset \Gamma_{h}\right\}$, in particular, $\mathcal{K}_{h}^{l}=\left\{K^{l}\right.$ : $\left.K \in \mathcal{K}_{h}^{l}\right\}$ is a partition of $\Gamma$.

Remark 3.1 The assumption that $\mathcal{T}_{h}$ is quasiuniform can be relaxed to locally quasiuniform meshes since all our arguments are local in the sense that elementwise or patchwise, with patches consisting of a uniformly bounded number of elements, estimates are used.

\subsection{The finite element method}

We let $V_{h}$ be the space of continuous piecewise linear functions defined on $\mathcal{T}_{h}$. The finite element method takes the form: find $u_{h} \in V_{h}$ such that

$$
A_{h}\left(u_{h}, v\right)=l_{h}(v) \quad \forall v \in V_{h}
$$

Here the forms are defined by

$$
A_{h}(v, w)=a_{h}(v, w)+j_{h}(v, w), \quad l_{h}(v)=(f, v)_{\Gamma_{h}}
$$

and

$$
\begin{aligned}
a_{h}(v, w) & =\left(\beta_{h} \cdot \nabla_{\Gamma_{h}} v, w\right)_{\Gamma_{h}}+\left(\alpha_{h} v, w\right)_{\Gamma_{h}} \\
j_{h}(v, w) & =c_{F} h\left(\left[n_{F} \cdot \nabla v\right],\left[n_{F} \cdot \nabla w\right]\right)_{\mathcal{F}_{h}}
\end{aligned}
$$

where $\nabla_{\Gamma_{h}} v=P_{\Gamma_{h}} \nabla v=\left(I-n_{h} \otimes n_{h}\right) \nabla v$ is the elementwise defined tangent gradient on $\Gamma_{h}, c_{F}$ is a positive stabilization parameter, $\alpha_{h}$ and $\beta_{h}$ are discrete approximations of $\alpha$ and $\beta$. The jump at a face $F$ shared by two elements $T^{+}$and $T^{-}$is defined by

$$
\left[n_{F} \cdot \nabla v\right]=n_{F}^{+} \cdot \nabla v^{+}+n_{F}^{-} \cdot \nabla v^{-}
$$


where $n_{F}^{ \pm}$is the exterior unit normal of the face $F$ and element $T^{ \pm}$and $v^{ \pm}=\left.v\right|_{T^{ \pm}}$.

In our forthcoming analysis we will need certain properties of the coefficients $\alpha_{h}$, $\beta_{h}$, and the right hand side $f_{h}$, in Sect. 5.2 we formulate the assumptions necessary for the stability analysis and in Sect. 6.1 we formulate the assumptions necessary for the a priori error estimates, and finally in Sect. 6.4 we provide a construction of discrete coefficients that satisfy all the assumptions.

\section{Preliminary results}

\subsection{Norms}

We let $\|v\|_{\omega}$ denote the $L^{2}$ norm over the set $\omega$ equipped with the appropriate Lebesgue measure. Furthermore, we introduce the scalar products

$$
\begin{aligned}
(v, w)_{\mathcal{T}_{h}} & =\sum_{T \in \mathcal{T}_{h}}(v, w)_{T}, \quad(v, w)_{\mathcal{K}_{h}}=\sum_{K \in \mathcal{K}_{h}}(v, w)_{K} \\
(v, w)_{\mathcal{F}_{h}} & =\sum_{F \in \mathcal{F}_{h}}(v, w)_{F}, \quad(v, w)_{\mathcal{E}_{h}}=\sum_{E \in \mathcal{E}_{h}}(v, w)_{E}
\end{aligned}
$$

with corresponding $L^{2}$ norms denoted by $\|\cdot\|_{\mathcal{T}_{h}},\|\cdot\|_{\mathcal{K}_{h}},\|\cdot\|_{\mathcal{F}_{h}}$, and $\|\cdot\|_{\mathcal{E}_{h}}$. Note that $\|\cdot\|_{\mathcal{K}_{h}}=\|\cdot\|_{\Gamma_{h}}$ and that the following scaling relations hold

$$
\sum_{T \in \mathcal{T}_{h}}|T| \sim h, \quad \sum_{K \in \mathcal{K}_{h}}|K| \sim \sum_{F \in \mathcal{F}_{h}}|F| \sim 1, \quad \sum_{E \in \mathcal{E}_{h}}|E| \sim h^{-1}
$$

Finally, we introduce the energy type norms

$$
\begin{aligned}
\|\| v \|_{h}^{2} & =\|v\|\left\|_{\mathcal{K}_{h}}^{2}+h\right\|\|v\| \|_{\mathcal{F}_{h}}^{2} \\
\|\| v\|\|_{\mathcal{K}_{h}}^{2} & =h\left\|\beta_{h} \cdot \nabla_{\Gamma_{h}} v\right\|_{\mathcal{K}_{h}}^{2}+\|v\|_{\mathcal{K}_{h}}^{2} \\
\|\| v\|\|_{\mathcal{F}_{h}}^{2} & =\left\|\left[n_{F} \cdot \nabla v\right]\right\|_{\mathcal{F}_{h}}^{2}
\end{aligned}
$$

\subsection{Inverse estimates}

Let $T \in \mathcal{T}_{h}, K=\Gamma_{h} \cap T, E \in \mathcal{E}_{h}$ and $E \subset \partial K$, then the following inverse estimates hold

$$
\begin{aligned}
& h\|v\|_{E}^{2} \lesssim\|v\|_{F}^{2} \quad \forall v \in V(F) \\
& h\|v\|_{F}^{2} \lesssim\|v\|_{T}^{2} \quad \forall v \in W(T) \\
& h\|v\|_{K}^{2} \lesssim\|v\|_{T}^{2} \quad \forall v \in W(T)
\end{aligned}
$$

with constants independent of the position of the intersection of $\Gamma_{h}$ and $T$. Note that the second inequality is the standard element to face inverse inequality. Here 
$V(F)=\widehat{V} \circ X_{F}^{-1}\left(W(T)=\widehat{W} \circ X_{T}^{-1}\right)$, where $\widehat{V}(\widehat{W})$ is a finite dimensional space on the reference triangle $\widehat{F}$ (reference tetrahedron $\widehat{T}$ ) and $X_{F}: \widehat{F} \rightarrow F\left(X_{T}: \widehat{T} \rightarrow T\right.$ ) an affine bijection.

\subsection{Extension and lifting of functions}

In this section we summarize basic results concerning extension and liftings of functions. We refer to $[6,11]$ for further details.

Extension Recalling the definition of the extension and using the chain rule we obtain the identity

$$
\nabla_{\Gamma_{h}} v^{e}=B^{T} \nabla_{\Gamma} v
$$

where

$$
B=P_{\Gamma}(I-\rho \mathcal{H}) P_{\Gamma_{h}}: T_{x}(K) \rightarrow T_{p(x)}(\Gamma)
$$

and $\mathcal{H}=\nabla \otimes \nabla \rho$. Here $\mathcal{H}$ is a $\Gamma$-tangential tensor, which equals the curvature tensor on $\Gamma$, and for small enough $\delta>0$, there is a constant such that

$$
\|\mathcal{H}\|_{L^{\infty}\left(U_{\delta}(\Gamma)\right)} \lesssim 1
$$

Furthermore, $B: T_{x}(K) \rightarrow T_{p(x)}(\Gamma)$ is invertible for $h \in\left(0, h_{0}\right]$ with $h_{0}$ small enough, i.e. there is $B^{-1}: T_{p(x)}(\Gamma) \rightarrow T_{x}(K)$ such that

$$
B B^{-1}=P, \quad B^{-1} B=P_{h}
$$

See [17] for further details.

Lifting The lifting $w^{l}$ of a function $w$ defined on $\Gamma_{h}$ to $\Gamma$ is defined as the push forward

$$
\left(w^{l}\right)^{e}=w^{l} \circ p=w \text { on } \Gamma_{h}
$$

and we have the identity

$$
\nabla_{\Gamma} w^{l}=B^{-T} \nabla_{\Gamma_{h}} w
$$

Estimates related to $B$ Using the uniform bound $\|\mathcal{H}\|_{L^{\infty}\left(U_{\delta}(\Gamma)\right)} \lesssim 1$, for $\delta>0$ small enough, it follows that

$$
\|B\|_{L^{\infty}\left(\Gamma_{h}\right)} \lesssim 1, \quad\left\|B^{-1}\right\|_{L^{\infty}(\Gamma)} \lesssim 1, \quad\left\|\left(P_{\Gamma}-B\right) P_{\Gamma_{h}}\right\|_{L^{\infty}\left(\Gamma_{h}\right)} \lesssim h^{2}
$$

Next consider the surface measure $d \Gamma=|B| d \Gamma_{h}$, where $|B|$ is the absolute value of the determinant of $\left[B \xi_{1} B \xi_{2} n^{e}\right]$ and $\left\{\xi_{1}, \xi_{2}\right\}$ is an orthonormal basis in $T_{x}(K)$. We have the following estimates

$$
\|1-|B|\|_{L^{\infty}\left(\Gamma_{h}\right)} \lesssim h^{2}, \quad\||B|\|_{L^{\infty}\left(\Gamma_{h}\right)} \lesssim 1, \quad\left\||B|^{-1}\right\|_{L^{\infty}\left(\Gamma_{h}\right)} \lesssim 1
$$

In view of these bounds and the identities (4.10) and (4.15) we obtain the following equivalences

$$
\left\|v^{l}\right\|_{L^{2}(\Gamma)} \sim\|v\|_{L^{2}\left(\Gamma_{h}\right)}, \quad\|v\|_{L^{2}(\Gamma)} \sim\left\|v^{e}\right\|_{L^{2}\left(\Gamma_{h}\right)}
$$


and

$$
\left\|\nabla_{\Gamma} v^{l}\right\|_{L^{2}(\Gamma)} \sim\left\|\nabla_{\Gamma_{h}} v\right\|_{L^{2}\left(\Gamma_{h}\right)}, \quad\left\|\nabla_{\Gamma} v\right\|_{L^{2}(\Gamma)} \sim\left\|\nabla_{\Gamma_{h}} v^{e}\right\|_{L^{2}\left(\Gamma_{h}\right)}
$$

\subsection{Interpolation}

Let $\pi_{h}: L^{2}\left(\mathcal{T}_{h}\right) \rightarrow V_{h}$ be the Clément interpolant. Then we have the following standard estimate

$$
\left\|v-\pi_{h} v\right\|_{H^{m}(T)} \lesssim h^{s-m}\|v\|_{H^{s}(\mathcal{N}(T))}, \quad m \leq s \leq 2, \quad m=0,1
$$

where $\mathcal{N}(T) \subset \mathcal{T}_{h}$ is the set of neighboring elements of $T$. In particular, we have the $L^{2}$ stability estimate

$$
\left\|\pi_{h} v\right\|_{T} \lesssim\|v\|_{\mathcal{N}(T)} \quad \forall T \in \mathcal{T}_{h}
$$

and as a consequence $\pi_{h}: L^{2}\left(\mathcal{T}_{h}\right) \rightarrow V_{h}$ is uniformly bounded and we have the estimate

$$
\left\|\pi_{h} v\right\|_{\mathcal{T}_{h}} \lesssim\|v\|_{\mathcal{T}_{h}}
$$

Using the trace inequality

$$
\|v\|_{T \cap \Gamma_{h}}^{2} \lesssim h^{-1}\|v\|_{T}^{2}+h\|\nabla v\|_{T}^{2}
$$

where the constant is independent of the position of the intersection between $\Gamma_{h}$ and $T$, see [16] for a proof, the interpolation inequality (4.20), and finally the stability of the extension operator

$$
\left\|v^{e}\right\|_{H^{s}\left(U_{\delta}(\Gamma)\right)} \lesssim \delta^{1 / 2}\|v\|_{H^{s}(\Gamma)} \quad 0<\delta \leq \delta_{0}
$$

with $\delta \sim h$, we obtain the interpolation error estimate

$$
\left\|v-\pi_{h} v\right\|_{H^{m}\left(\mathcal{K}_{h}\right)} \lesssim h^{s-m}\|v\|_{H^{s}(\Gamma)} \quad m \leq s \leq 2, \quad m=0,1
$$

Using (4.25) and the definition of the energy norm (4.5) we obtain

$$
\left\|\left.\left|v^{e}-\pi_{h} v^{e}\right|\right|_{\mathcal{K}_{h}} \lesssim h^{3 / 2}\right\| v \|_{H^{2}(\Gamma)}
$$

and using a standard trace inequality on tetrahedra, the interpolation estimate (4.20), and the stability (4.24) of the extension, we have

$$
\left\|\left|v^{e}-\pi_{h} v^{e}\right|\right\| \mathcal{F}_{h} \lesssim h\|v\|_{H^{2}(\Gamma)}
$$

Combining these two estimates we get

$$
\left\|\left|v^{e}-\pi_{h} v^{e}\right|\right\|_{h} \lesssim h^{3 / 2}\|v\|_{H^{2}(\Gamma)}
$$




\section{Stability estimates}

\subsection{Coverings}

In this section we begin by recalling a construction of coverings of $\mathcal{T}_{h}$ developed in [6], Sect. 4.1. The number of tetrahedra in the covering sets are uniformly bounded and the area of their intersection with $\Gamma_{h}$ is equivalent to $h^{2}$. See also [12] for related results. Then we formulate two useful lemmas.

Families of coverings of $\mathcal{T}_{h}$ Let $x$ be a point on $\Gamma$ and let $B_{\delta}(x)=\left\{y \in \mathbb{R}^{3}:|y-x|<\right.$ $\delta\}$ and $D_{\delta}(x)=B_{\delta}(x) \cap \Gamma$. We define the sets of elements

$$
\mathcal{K}_{\delta, x}=\left\{K \in \mathcal{K}_{h}: \bar{K}^{l} \cap D_{\delta}(x) \neq \emptyset\right\}, \quad \mathcal{T}_{\delta, x}=\left\{T \in \mathcal{T}_{h}: T \cap \Gamma_{h} \in \mathcal{K}_{\delta, x}\right\}
$$

With $\delta \sim h$ we use the notation $\mathcal{K}_{h, x}$ and $\mathcal{T}_{h, x}$. For each $\mathcal{T}_{h}, h \in\left(0, h_{0}\right]$ there is a set of points $\mathcal{X}_{h}$ on $\Gamma$ such that $\left\{\mathcal{K}_{h, x}, x \in \mathcal{X}_{h}\right\}$ and $\left\{\mathcal{T}_{h, x}, x \in \mathcal{X}_{h}\right\}$ are coverings of $\mathcal{T}_{h}$ and $\mathcal{K}_{h}$ with the following properties:

- The number of sets containing a given point $y$ is uniformly bounded

$$
\#\left\{x \in \mathcal{X}_{h}: y \in \mathcal{T}_{h, x}\right\} \lesssim 1 \quad \forall y \in \mathbb{R}^{3}
$$

for all $h \in\left(0, h_{0}\right]$ with $h_{0}$ small enough.

- The number of elements in the sets $\mathcal{T}_{h, x}$ is uniformly bounded

$$
\# \mathcal{T}_{h, x} \lesssim 1 \quad \forall x \in \mathcal{X}_{h}
$$

for all $h \in\left(0, h_{0}\right]$ with $h_{0}$ small enough, and each element in $\mathcal{T}_{h, x}$ share at least one face with another element in $\mathcal{T}_{h, x}$.

- $\forall h \in\left(0, h_{0}\right]$ and $\forall x \in \mathcal{X}_{h}, \exists T_{x} \in \mathcal{T}_{h, x}$ that has a large intersection with $\Gamma_{h}$ in the sense that

$$
\left|T_{x} \cap \Gamma_{h}\right|=\left|K_{x}\right| \sim h^{2} \quad \forall x \in \mathcal{X}_{h},
$$

for all $h \in\left(0, h_{0}\right]$ with $h_{0}$ small enough.

We first recall a Lemma from [6] and then we prove a lemma tailored to the particular demands of this paper.

Lemma 5.1 It holds

$$
\|v\|_{\mathcal{T}_{h}}^{2} \lesssim h\left(\|v\|_{\mathcal{K}_{h}}^{2}+\|\| v \|_{\mathcal{F}_{h}}^{2}\right) \quad \forall v \in V_{h}
$$

for all $h \in\left(0, h_{0}\right]$ with $h_{0}$ small enough.

Proof See Lemma 4.5 in [6].

Lemma 5.2 It holds

$$
h\|v\|_{\mathcal{E}_{h}}^{2} \lesssim\|v\|_{\mathcal{K}_{h}}^{2}+h^{2}\|\| v \|_{\mathcal{F}_{h}}^{2} \quad \forall v \in V_{h}
$$

for all $h \in\left(0, h_{0}\right]$ with $h_{0}$ small enough. 
Proof Consider an arbitrary set in the covering described above. Then we shall prove that we have the estimate

$$
h\|v\|_{\mathcal{E}_{h, x}}^{2} \lesssim\|v\|_{\mathcal{K}_{h, x}}^{2}+h^{2}\left\|\left[n_{F} \cdot \nabla v\right]\right\|_{\mathcal{F}_{h, x}}^{2}
$$

where $\mathcal{F}_{h, x}$ is the set of interior faces in $\mathcal{T}_{h, x}$. Let $v_{x}: \mathcal{T}_{h, x} \rightarrow \mathbb{R}$ be the first order polynomial that satisfies $v_{x}=\left.v\right|_{T_{x}}$, where $T_{x}$ is the element with a large intersection $K_{x}$. Adding and subtracting $v_{x}$ we get

$$
h\|v\|_{\mathcal{E}_{h, x}}^{2} \leq h\left\|v-v_{x}\right\|_{\mathcal{E}_{h, x}}^{2}+h\left\|v_{x}\right\|_{\mathcal{E}_{h, x}}^{2}=I+I I
$$

Term I We have

$$
h\left\|v-v_{x}\right\|_{\mathcal{E}_{h, x}}^{2} \lesssim h^{-1}\left\|v-v_{x}\right\|_{\mathcal{T}_{h, x}}^{2} \lesssim h^{2}\|\| v \|_{\mathcal{F}_{h, x}}^{2}
$$

where we used the inverse estimates (4.7) and (4.8) to pass from $\mathcal{E}_{h}$ to $\mathcal{T}_{h}$, the inequality

$$
\|w\|_{\mathcal{T}_{h, x}}^{2} \lesssim\|w\|_{T_{x}}^{2}+\left.h^{3}\||w|\|_{\mathcal{F}_{h, x}}^{2} \quad \forall w \in V_{h}\right|_{\mathcal{T}_{h, x}}
$$

with $w=v-v_{x}=0$ on $T_{x}$, and finally the fact that $\left[n_{F} \cdot \nabla v_{x}\right]=0$.

Verification of (5.10) Considering a pair of elements $T_{1}, T_{2} \in \mathcal{T}_{h, x}$ that share a face $F$, with center of gravity $x_{F}$, we have the identity

$$
w_{2}(x)=w_{1}(x)+\left.\left[n_{F} \cdot \nabla w\right]\right|_{x_{F}}\left(x-x_{F}\right) \cdot n_{F}, \quad x \in T_{1} \cup T_{2}
$$

where $w$ is a continuous piecewise linear polynomial on $T_{1} \cup T_{2}$ and $w_{i}$ the linear polynomial on $T_{1} \cup T_{2}$ such that $\left.w_{i}\right|_{T_{i}}=\left.w\right|_{T_{i}}, i=1$, 2. Integrating over $T_{2}$ gives

$$
\begin{aligned}
\left\|w_{2}\right\|_{T_{2}}^{2} & \lesssim \underbrace{\left\|w_{1}\right\|_{T_{2}}^{2}}_{I_{1}}+\underbrace{\left\|\left.\left[n_{F} \cdot \nabla w\right]\right|_{x_{F}}\left(x-x_{F}\right) \cdot n_{F}\right\|_{T_{2}}^{2}}_{I_{2}} \\
& \lesssim\left\|w_{1}\right\|_{T_{1}}^{2}+h^{3}\left\|\left[n_{F} \cdot \nabla w\right]\right\|_{F}^{2}
\end{aligned}
$$

To estimate $I_{1}$ we used the inverse inequality

$$
\left\|w_{1}\right\|_{T_{2}} \lesssim\left\|w_{1}\right\|_{T_{1}}
$$

which we may prove by letting $G_{1}: \mathbb{R}^{3} \rightarrow \mathbb{R}^{3}$ be the affine mapping which maps the reference tetrahedron $\widehat{T}$ onto $T_{1}$. We note using shape regularity that there is a ball $B_{R}\left(\widehat{x}_{\widehat{T}}\right)$ of radius $R \lesssim 1$ centered at the center of gravity $\widehat{x}_{\widehat{T}}$ of $\widehat{T}$ such that $G_{1}^{-1}\left(T_{2}\right) \subset B_{R}\left(\widehat{x}_{\widehat{T}}\right)$. Changing domain of integration and using an inverse bound on the reference configuration we obtain 


$$
\begin{aligned}
\|v\|_{T_{2}}^{2} & =\int_{G_{1}^{-1}\left(T_{2}\right)}\left|v \circ G_{1}\right|^{2}\left|D G_{1}\right| \leq \int_{B_{R}\left(\widehat{x}_{\widehat{T}}\right)}\left|v \circ G_{1}\right|^{2}\left|D G_{1}\right| \\
& \lesssim \int_{\widehat{T}}\left|v \circ G_{1}\right|^{2}\left|D G_{1}\right|=\int_{G_{1}(\widehat{T})}|v|^{2}\left|D G_{1}\right|\left|D G_{1}^{-1}\right|=\|v\|_{T_{1}}^{2}
\end{aligned}
$$

where $\left|D G_{1}\right|$ is the absolute value of the determinant of the derivative of $G_{1}$ which is constant since $G_{1}$ is affine.

To estimate $I_{2}$ we used the facts that $\left[n_{F} \cdot \nabla v\right]$ is constant on $F$ and that $z=$ $\left(x-x_{F}\right) \cdot n_{F}$ is the signed distance from $x$ to $F$ which satisfies $|z| \leq C h$. We then have

$$
\left\|\left.\left[n_{F} \cdot \nabla w\right]\right|_{x_{F}}\left(x-x_{F}\right) \cdot n_{F}\right\|_{T_{2}}^{2} \leq\left.\int_{F} \int_{0}^{C h}\left|\left[n_{F} \cdot \nabla w\right]\right| x_{F}\right|^{2} z^{2} \lesssim h^{3}\left\|\left[n_{F} \cdot \nabla w\right]\right\|_{F}^{2}
$$

Finally, iterating (5.13) and using the fact that the number of elements in $\mathcal{T}_{h, x}$ is uniformly bounded (5.10) follows.

Term II We first split $v_{x}$ into one term $v_{x, c}$ which is constant in the direction normal to $K_{x}$ and a reminder term $v_{x}-v_{x, c}=t n_{x} \cdot \nabla v_{x}$ where $n_{x}=\left.n_{h}\right|_{K_{x}}$ and $t$ is the signed distance to the hyperplane in which $K_{x}$ is contained, as follows

$$
v_{x}=v_{x, c}+t n_{x} \cdot \nabla v_{x}
$$

Using the triangle inequality we obtain

$$
h\left\|v_{x}\right\|_{\mathcal{E}_{h, x}}^{2} \lesssim h\left\|v_{x, c}\right\|_{\mathcal{E}_{h, x}}^{2}+h\left\|t n_{x} \cdot \nabla v\right\|_{\mathcal{E}_{h, x}}^{2}=I I_{1}+I I_{2}
$$

Term $I I_{1}$ We have

$$
I I_{1}=h\left\|v_{x, c}\right\|_{\mathcal{E}_{h, x}}^{2} \lesssim h^{-1}\left\|v_{x, c}\right\|_{\mathcal{T}_{h, x}}^{2} \lesssim h^{-1}\left\|v_{x, c}\right\|_{T_{x}}^{2} \lesssim\left\|v_{x, c}\right\|_{K_{x}}^{2} \lesssim\|v\|_{\mathcal{K}_{h, x}}^{2}
$$

where we used the inverse estimates (4.7) and (4.8) to pass from $\mathcal{E}_{h}$ to $\mathcal{T}_{h}$, an inverse estimate using the fact that $v_{x, c}$ is a polynomial on $\mathcal{T}_{h, x}$, finally an inverse inequality which holds since $v_{x, c}$ is constant in the normal direction.

Term $I_{2}$ We have

$$
\begin{aligned}
I I_{2} & \lesssim h\|t\|_{L^{\infty}\left(\mathcal{E}_{h, x}\right)}^{2}\left\|n_{x} \cdot \nabla v_{x}\right\|_{\mathcal{E}_{h, x}}^{2} \lesssim h^{5}\left\|\nabla v_{x}\right\|_{\mathcal{E}_{h, x}}^{2} \\
& \lesssim h^{3}\left\|\nabla v_{x}\right\|_{T_{x}}^{2} \lesssim h\left\|v_{x}\right\|_{T_{x}}^{2} \lesssim h\|v\|_{\mathcal{T}_{h, x}}^{2}
\end{aligned}
$$

where we used Hölder's inequality, the bound

$$
\|t\|_{L^{\infty}\left(\mathcal{E}_{h, x}\right)} \lesssim h^{2}
$$

the inverse estimates (4.7) and (4.8) to pass from $\mathcal{E}_{h}$ to $\mathcal{T}_{h}$, an inverse inequality to pass from $H^{1}$ to $L^{2}$, and finally the fact that $v_{x}=v$ on $T_{x} \in \mathcal{T}_{h, x}$. 
Verification of (5.22) We note that each point $y \in \mathcal{K}_{h, x}$ can be connected to a point $z \in K_{x}$ using a piecewise linear curve in $\mathcal{K}_{h, x}$ consisting of a finite number of segments, each residing in a facet $K_{i} \in \mathcal{K}_{h, x}$, of the form

$$
y=z+\sum_{i=1}^{N} s_{i} a_{i}
$$

where $0 \leq s_{i} \lesssim h$ is the arclength parameter of each segment, $a_{i} \in T\left(K_{i}\right)$ is a unit direction vector in the tangent space $T\left(K_{i}\right)$ to $K_{i}$, and $N$ is uniformly bounded. Then $t(y)=n_{x} \cdot(y-z)$ and we have the estimate

$$
|t(y)| \leq \sum_{i=1}^{N}\left|s_{i}\right|\left|n_{x} \cdot a_{i}\right| \leq \sum_{i=1}^{N}\left|s_{i}\right|\left|\left(n_{x}-n_{h, i}\right) \cdot a_{i}\right| \leq \sum_{i=1}^{N}\left|s_{i}\right|\left|n_{x}-n_{h, i}\right| \lesssim h^{2}
$$

where $n_{h, i}$ is the normal to the facet in which the $i$ :th segment reside and we used the estimate $\left|n_{x}-n_{h, i}\right| \lesssim h$, which follows from the fact that at each edge $E$ shared by two facets $K_{1}, K_{2} \in \mathcal{K}_{h, x}$ with normals $n_{h, 1}$ and $n_{h, 2}$ we have the estimate

$$
\left|n_{h, 1}-n_{h, 2}\right| \leq\left|n_{h, 1}-n\right|+\left|n-n_{h, 2}\right| \lesssim h
$$

and thus we obtain the bound since the number of elements in $\mathcal{K}_{h, x}$ is uniformly bounded.

Conclusion Collecting the estimates we obtain

$$
h\|v\|_{\mathcal{E}_{h, x}}^{2} \lesssim I+I I_{1}+I I_{2} \lesssim\|v\|_{\mathcal{K}_{h, x}}^{2}+h^{2}\|\| v\left\|_{\mathcal{F}_{h, x}}^{2}+h\right\| v \|_{\mathcal{T}_{h, x}}^{2}
$$

Summing over the covering and using Lemma 5.1 gives

$$
\begin{aligned}
h\|v\|_{\mathcal{E}_{h}}^{2} & \lesssim\|v\|_{\mathcal{K}_{h}}^{2}+h^{2} \mid\|v\|\left\|_{\mathcal{F}_{h}}^{2}+h\right\| v \|_{\mathcal{T}_{h}}^{2} \\
& \lesssim\|v\|_{\mathcal{K}_{h}}^{2}+h^{2} \mid\|v\|_{\mathcal{F}_{h}}^{2}+h^{2}\left(\|v\|_{\mathcal{K}_{h}}^{2}+\|\| v \|\left.\right|_{\mathcal{F}_{h}} ^{2}\right) \\
& \lesssim\|v\|_{\mathcal{K}_{h}}^{2}+h^{2} \mid\|v\|_{\mathcal{F}_{h}}^{2}
\end{aligned}
$$

which concludes the proof.

\subsection{Assumptions on the coefficients for the stability estimates}

In order to prove the stability estimates we make the following assumptions on the coefficients.

Assumption We assume that the discrete coefficients $\alpha_{h}$ and $\beta_{h}$ are uniformly bounded and satisfy: 
- There is a constant such that

$$
0<C \leq \inf _{x \in \Gamma_{h}}\left(\alpha_{h}(x)-\frac{1}{2} \operatorname{div}_{\Gamma_{h}} \beta_{h}(x)\right)
$$

- For $x \in \mathcal{X}_{h}$, there is a constant vector field $\beta_{h, x}: \mathcal{K}_{h, x} \rightarrow \mathbb{R}^{3}$ such that

$$
\left\|\beta_{h}-\beta_{h, x}\right\|_{L^{\infty}\left(\mathcal{K}_{h, x}\right)} \leq C h
$$

where the constant is independent of $x \in \mathcal{X}_{h}$.

- There is a constant such that

$$
\left\|\left[n_{E} \cdot \beta_{h}\right]\right\|_{L^{\infty}\left(\mathcal{E}_{h}\right)} \leq C h^{2}
$$

for all $h \in\left(0, h_{0}\right]$ with $h_{0}$ small enough. The jump at an edge $E$ shared by two surface elements $K^{ \pm}$is defined by

$$
\left[n_{E} \cdot \beta_{h}\right]=n_{E}^{+} \cdot \beta_{h}^{+}+n_{E}^{-} \cdot \beta_{h}^{-}
$$

where $n_{E}^{ \pm}$is the unit exterior conormal to $K^{ \pm}$associated with edge $E$, i.e. the unique unit vector that is normal to $E$ and tangent and exterior to $K^{ \pm}$, and $\beta_{h}^{ \pm}=\left.\beta_{h}\right|_{K^{ \pm}}$.

We return to a construction of $\alpha_{h}$ and $\beta_{h}$ in Sect. 6.4. Anticipating the forthcoming analysis we mention that a sufficent condition for the construction of $\alpha_{h}$ and $\beta_{h}$ satisfying (5.30-5.32) as well as the necessary data approximation estimates, see Sect. 6.1, is that $\alpha \in C^{2}(\Gamma)$ and $\beta \in C^{2}(\Gamma)$.

\subsection{Technical lemmas}

Lemma 5.3 There is a constant such that

$$
\left\|\left(I-\pi_{h}\right)\left(\beta_{h} \cdot \nabla_{\Gamma_{h}} v\right)\right\|_{\mathcal{K}_{h}}^{2} \lesssim\|v\|_{\mathcal{K}_{h}}^{2}+\|\| v \|_{\mathcal{F}_{h}}^{2} \quad \forall v \in V_{h}
$$

for all $h \in\left(0, h_{0}\right]$ with $h_{0}$ small enough.

Proof We use a covering $\left\{\mathcal{T}_{h, x}: x \in \mathcal{X}_{h}\right\}$ as described above and we introduced the notation

$$
\mathcal{N}\left(\mathcal{T}_{h, x}\right)=\mathcal{N}\left(\mathcal{T}_{h, x}\right)
$$

for the set of elements $T \in \mathcal{T}_{h}$ that are neighbors to an element in $\mathcal{T}_{h, x}$. i.e. share at least one vertex with an element in $\mathcal{T}_{h, x}$ and $\mathcal{F}_{h}\left(\mathcal{N}\left(\mathcal{T}_{h, x}\right)\right)$ for the set of interior faces in $\mathcal{N}\left(\mathcal{T}_{h, x}\right)$. The larger set $\mathcal{N}\left(\mathcal{T}_{h, x}\right)$ naturally occurs when we employ the $L^{2}$ stability (4.21) of the interpolation operator. We then have 


$$
\begin{aligned}
h \|(I & \left.-\pi_{h}\right)\left(\beta_{h} \cdot \nabla_{\Gamma_{h}} v\right) \|_{\mathcal{K}_{h, x}}^{2} \\
& \lesssim\left\|\left(I-\pi_{h}\right)\left(\beta_{h} \cdot \nabla_{\Gamma_{h}} v\right)\right\|_{\mathcal{T}_{h, x}}^{2} \\
& \lesssim\left\|\left(I-\pi_{h}\right)\left(\beta_{h, x} \cdot \nabla v\right)\right\|_{\mathcal{T}_{h, x}}^{2}+\left\|\left(I-\pi_{h}\right)\left(\left(\beta_{h}-\beta_{h, x}\right) \cdot \nabla v\right)\right\|_{\mathcal{T}_{h, x}}^{2} \\
& \lesssim h\left\|\left[n_{F} \cdot \nabla v\right]\right\|_{\mathcal{F}_{h}\left(\mathcal{N}\left(\mathcal{T}_{h, x}\right)\right)}^{2}+\left\|\beta_{h}-\beta_{h, x}\right\|_{L^{\infty}\left(\mathcal{N}\left(\mathcal{T}_{h, x}\right)\right)}^{2}\|\nabla v\|_{\mathcal{N}\left(\mathcal{T}_{h, x}\right)}^{2} \\
& \lesssim h\left\|\left[n_{F} \cdot \nabla v\right]\right\|_{\mathcal{F}_{h}\left(\mathcal{N}\left(\mathcal{T}_{h, x}\right)\right)}^{2}+\|v\|_{\mathcal{N}\left(\mathcal{T}_{h, x}\right)}^{2}
\end{aligned}
$$

where we used the following estimates. (5.36): An inverse bound to pass from $\mathcal{K}_{h, x}$ to $\mathcal{T}_{h, x}$. (5.37): Added and subtracted the constant vector field $\beta_{h, x}$, defined in (5.31), and used the triangle inequality. (5.38): The second term on the right hand side of (5.37) is estimated using $L^{2}$ stability (4.21) of $\pi_{h}$ and Hölder's inequality and for the first term we used the following Poincaré inequality

$$
\left\|\left(I-\pi_{h}\right) w\right\|_{\mathcal{T}_{h, x}}^{2} \lesssim h\|[w]\|_{\mathcal{F}_{h}\left(\mathcal{N}\left(\mathcal{T}_{h, x}\right)\right)}^{2} \quad \forall w \in D P_{0}\left(\mathcal{N}\left(\mathcal{T}_{h, x}\right)\right)
$$

where $D P_{0}\left(\mathcal{N}\left(\mathcal{T}_{h, x}\right)\right)$ is the space of piecewise constant functions on $\mathcal{N}\left(\mathcal{T}_{h, x}\right)$, followed by the estimate $\left\|\left[\beta_{h, x} \cdot \nabla v\right]\right\|_{F} \leq\left\|\beta_{h, x}\right\|_{L^{\infty}(F)} \|\left[\nabla v\left\|_{F} \lesssim\right\|\left[n_{F} \cdot \nabla v\right] \|_{F} \cdot\right.$. (5.39): The bound (5.31) followed by an inverse estimate to remove the gradient.

Verification of (5.40) For each element $T \in \mathcal{T}_{h, x}$, we shall prove that

$$
\left\|\left(I-\pi_{h}\right) w\right\|_{T}^{2} \lesssim\|[w]\|_{\mathcal{F}_{h}(\mathcal{N}(T))}^{2}
$$

Let $w_{T}$ be the constant function on $\mathcal{N}(T)$ such that $\left.w_{T}\right|_{T}=\left.w\right|_{T}$, then we have

$$
\left\|\left(I-\pi_{h}\right) w\right\|_{T}=\left\|\left(I-\pi_{h}\right)\left(w-w_{T}\right)\right\|_{T} \lesssim\left\|w-w_{T}\right\|_{\mathcal{N}(T)}^{2}
$$

where we used $L^{2}$ stability (4.21) of $\pi_{h}$. Now for any element $T^{\prime} \in \mathcal{N}(T)$ there is finite chain of elements from $T$ to $T^{\prime}$ that are face neighbors and we clearly have the estimate

$$
\left\|w-w_{T}\right\|_{T^{\prime}}^{2} \lesssim h\|[w]\|_{\mathcal{F}_{h}(\mathcal{N}(T))}^{2}
$$

since $w-w_{T}=0$ on $T$. Summing over all elements $T^{\prime} \in \mathcal{N}(T)$, estimate (5.42) gives

$$
\left\|\left(I-\pi_{h}\right) w\right\|_{T} \lesssim\left\|w-w_{T}\right\|_{\mathcal{N}(T)}^{2} \lesssim h\|[w]\|_{\mathcal{F}_{h}(\mathcal{N}(T))}^{2}
$$

Finally, summing over all $T \in \mathcal{T}_{h, x}$, estimate (5.44) gives the desired estimate (5.40).

Conclusion Finally, summing over the covering sets and using Lemma 5.1 we obtain

$$
\begin{aligned}
h\left\|\left(I-\pi_{h}\right)\left(\beta_{h} \cdot \nabla_{\Gamma_{h}} v\right)\right\|_{\mathcal{K}_{h}}^{2} & \lesssim\|v\|_{\mathcal{T}_{h}}^{2}+h\left\|\left[n_{F} \cdot \nabla v\right]\right\|_{\mathcal{F}_{h}}^{2} \\
& \lesssim h\|v\|_{\mathcal{K}_{h}}^{2}+h\left\|\left[n_{F} \cdot \nabla u\right]\right\|_{\mathcal{F}_{h}}^{2}
\end{aligned}
$$

which concludes the proof. 
Lemma 5.4 There is a constant such that

$$
\left\|\beta_{h} \cdot \nabla_{\Gamma_{h}} v\right\|_{\mathcal{T}_{h}}^{2} \lesssim h\|v\|_{\mathcal{K}_{h}}^{2}+h\left\|\beta_{h} \cdot \nabla_{\Gamma_{h}} v\right\|_{\mathcal{K}_{h}}^{2}+h\|v\| \|_{\mathcal{F}_{h}}^{2} \quad \forall v \in V_{h}
$$

for all $h \in\left(0, h_{0}\right]$ with $h_{0}$ small enough.

Proof We again consider an arbitrary set $\mathcal{T}_{h, x}$ in the covering. Adding and subtracting $\beta_{h, x}$, that satisfies the estimate (5.31), and using the triangle inequality we get

$$
\begin{aligned}
\left\|\beta_{h} \cdot \nabla_{\Gamma_{h}} v\right\|_{\mathcal{T}_{h, x}}^{2} & \leq\left\|\beta_{h, x} \cdot \nabla_{\Gamma_{h}} v\right\|_{\mathcal{T}_{h, x}}^{2}+\left\|\left(\beta_{h}-\beta_{h, x}\right) \cdot \nabla_{\Gamma_{h}} v\right\|_{\mathcal{T}_{h, x}}^{2} \\
& \lesssim\left(h\left\|\beta_{h, x} \cdot \nabla_{\Gamma_{h}} v\right\|_{K_{x}}^{2}+h\|\| v \|_{\mathcal{F}_{h, x}}^{2}\right)+h^{2}\|\nabla v\|_{\mathcal{T}_{h, x}}^{2} \\
& \lesssim\left(h\left\|\beta_{h, x} \cdot \nabla_{\Gamma_{h}} v\right\|_{\mathcal{K}_{h, x}}^{2}+h\|v\| \|_{\mathcal{F}_{h, x}}^{2}\right)+\|v\|_{\mathcal{T}_{h, x}}^{2}
\end{aligned}
$$

Here we used the following estimates: (5.49): Again using an argument, similar to the verification of (5.40), we conclude that

$$
\|w\|_{\mathcal{T}_{h, x}}^{2} \lesssim\|w\|_{T}^{2}+h\|[w]\|_{\mathcal{F}_{h, x}}^{2} \quad \forall w \in D P_{0}\left(\mathcal{T}_{h, x}\right)
$$

for any $T \in \mathcal{T}_{h, x}$. Now taking $T=T_{x}$, the element with a large intersection $T \cap \Gamma_{h}$ we also have the inverse estimate $\|w\|_{T_{x}}^{2} \lesssim h\|w\|_{K_{x}}^{2}$ since $w$ is constant on $T$. (5.50): Follows directly from the fact that $K_{x} \in \mathcal{K}_{h, x}$.

Summing over the sets in the covering gives

$$
\left\|\beta_{h} \cdot \nabla_{\Gamma_{h}} v\right\|_{\mathcal{T}_{h}}^{2} \lesssim h\left\|\beta_{h} \cdot \nabla_{\Gamma_{h}} v\right\|_{\mathcal{K}_{h}}^{2}+h\|\| v\left\|_{\mathcal{F}_{h}}^{2}+\right\| v \|_{\mathcal{T}_{h}}^{2}
$$

and using Lemma 5.1 we can bound the last term and arrive at

$$
\left\|\beta_{h} \cdot \nabla_{\Gamma_{h}} v\right\|_{\mathcal{T}_{h}}^{2} \lesssim h\left\|\beta_{h} \cdot \nabla_{\Gamma_{h}} v\right\|_{\mathcal{K}_{h}}^{2}+h\|\| v\left\|_{\mathcal{F}_{h}}^{2}+h\right\| v \|_{\mathcal{K}_{h}}^{2}
$$

which concludes the proof.

\subsection{Stability estimates}

Lemma 5.5 There is a positive constant $m_{0}$ such that

$$
m_{0}\left(\|v\|_{\mathcal{K}_{h}}^{2}+h \mid\|v\| \|_{\mathcal{F}_{h}}^{2}\right) \leq A_{h}(v, v) \quad \forall v \in V_{h}
$$

for all $h \in\left(0, h_{0}\right]$ with $h_{0}$ small enough.

Proof Integrating by parts elementwise over the surface mesh $\mathcal{K}_{h}$ we obtain the identity

$$
2\left(\beta_{h} \cdot \nabla_{\Gamma_{h}} v, v\right)_{\mathcal{K}_{h}}=\left(\left[n_{E} \cdot \beta_{h}\right] v, v\right)_{\mathcal{E}_{h}}-\left(\left(\nabla_{\Gamma_{h}} \cdot \beta_{h}\right) v, v\right)_{\mathcal{K}_{h}}
$$


The first term on the right hand side may be estimated as follows

$$
\begin{aligned}
\left(\left[n_{E} \cdot \beta_{h}\right] v, v\right)_{\mathcal{E}_{h}} & =\left\|\left[n_{E} \cdot \beta_{h}\right]\right\|_{L^{\infty}\left(\mathcal{E}_{h}\right)}\|v\|_{\mathcal{E}_{h}}^{2} \\
& \lesssim h^{2}\|v\|_{\mathcal{E}_{h}}^{2} \\
& \lesssim h\|v\|_{\mathcal{K}_{h}}^{2}+h^{3}\|\| v\|\|_{\mathcal{F}_{h}}^{2}
\end{aligned}
$$

where we used Hölder's inequality, the assumption (5.32) on $\beta_{h}$, and at last Lemma 5.2. Thus we arrive at the estimate

$$
\left|\left(\left[n_{E} \cdot \beta_{h}\right] v, v\right)_{\mathcal{E}_{h}}\right| \leq C_{\star} h\left(\|v\|_{\mathcal{K}_{h}}^{2}+h^{2} \mid\|v\| \|_{\mathcal{F}_{h}}^{2}\right)
$$

We now have

$$
\begin{aligned}
A_{h}(v, v)= & \left(\beta_{h} \cdot \nabla_{\Gamma_{h}} v, v\right)_{\Gamma_{h}}+\left(\alpha_{h} v, v\right)_{\Gamma_{h}}+\left(c_{F} h\left[n_{F} \cdot \nabla v\right],\left[n_{F} \cdot \nabla v\right]\right)_{\mathcal{F}_{h}} \\
= & \left(\left(\alpha_{h}-2^{-1} \operatorname{div}_{\Gamma_{h}} \beta_{h}\right) v, v\right)_{\Gamma_{h}} \\
& \quad+2^{-1}\left(\left[n_{E} \cdot \beta_{h}\right] v, v\right)_{\mathcal{E}_{h}}+\left(c_{F} h\left[n_{F} \cdot \nabla v\right],\left[n_{F} \cdot \nabla v\right] \mathcal{F}_{h}\right. \\
\geq & \inf _{\mathcal{K}_{h}}\left(\alpha_{h}-2^{-1} \operatorname{div}_{\Gamma_{h}} \beta_{h}\right)\|v\|_{\mathcal{K}_{h}}^{2} \\
& \quad-2^{-1} C_{\star} h\left(\|v\|_{\mathcal{K}_{h}}^{2}+h^{2}\|v\|_{\mathcal{F}_{h}}^{2}\right)+c_{F} h\|v\|_{\mathcal{F}_{h}}^{2} \\
\geq & \inf \left(\alpha_{h}-2^{-1} \operatorname{div}_{\Gamma_{h}} \beta_{h}-2^{-1} C_{\star} h\right)\|v\|_{\mathcal{K}_{h}}^{2} \\
& \quad+\min \left(c_{F}-2^{-1} C_{\star} h^{2}\right) h\|v\|_{\mathcal{F}_{h}}^{2}
\end{aligned}
$$

where we used the identity (5.55), the estimate (5.59), and then we collected the terms. Thus we find that

$$
\|v\|_{\mathcal{K}_{h}}^{2}+h\left\|\left[n_{F} \cdot \nabla v\right]\right\|_{\mathcal{F}_{h}}^{2} \lesssim A_{h}(v, v)
$$

for $c_{F}>0$ and $h \in\left(0, h_{0}\right]$ with $h_{0}$ small enough.

Lemma 5.6 There are positive constants $m_{1}$ and $m_{2}$ such that

$$
m_{1} h\left\|\beta_{h} \cdot \nabla_{\Gamma_{h}} v\right\|_{\mathcal{K}_{h}}^{2}-m_{2} A_{h}(v, v) \leq A_{h}\left(v, h \pi_{h}\left(\beta_{h} \cdot \nabla_{\Gamma_{h}} v\right)\right) \quad v \in V_{h}
$$

for all $h \in\left(0, h_{0}\right]$ with $h_{0}$ small enough.

Proof We have

$$
\begin{aligned}
A_{h}\left(v, h \pi_{h}\left(\beta_{h} \cdot \nabla_{\Gamma_{h}} v\right)\right)= & \left(\beta_{h} \cdot \nabla_{\Gamma_{h}} v, h \beta_{h} \cdot \nabla_{\Gamma_{h}} v\right) \mathcal{K}_{h} \\
& \underbrace{-\left(\beta_{h} \cdot \nabla_{\Gamma_{h}} v, h\left(I-\pi_{h}\right)\left(\beta_{h} \cdot \nabla_{\Gamma_{h}} v\right)\right) \mathcal{K}_{h}}_{I} \\
& \underbrace{+\left(\alpha_{h} v, h \pi_{h}\left(\beta_{h} \cdot \nabla_{\Gamma_{h}} v\right)\right) \mathcal{K}_{h}}_{I I}
\end{aligned}
$$




$$
\begin{gathered}
\underbrace{+j_{h}\left(v, h \pi_{h}\left(\beta_{h} \cdot \nabla_{\Gamma_{h}} v\right)\right.}_{I I I} \\
\geq h\left\|\beta_{h} \cdot \nabla_{\Gamma_{h}} v\right\|_{\mathcal{K}_{h}}^{2}-|I+I I+I I I|
\end{gathered}
$$

We now have the estimate

$$
|I+I I+I I I| \leq C_{1}\left(\delta+\delta^{-1}\right) A_{h}(v, v)+C_{2} \delta h\left\|\beta_{h} \cdot \nabla_{\Gamma_{h}} v\right\|_{\mathcal{K}_{h}}^{2}
$$

for $\delta>0$. Thus taking $\delta$ small enough the desired estimate follows directly by combining (5.67) and (5.68).

Verification of (5.68) The estimate follows by combining the following estimates of Terms $I-I I I$.

Term I. It holds

$$
\begin{aligned}
|I| & \lesssim \delta h\left\|\beta_{h} \cdot \nabla_{\Gamma_{h}} v\right\|_{\mathcal{K}_{h}}^{2}+\delta^{-1} h\left\|\left(I-\pi_{h}\right) \beta_{h} \cdot \nabla_{\Gamma_{h}} v\right\|_{\mathcal{K}_{h}}^{2} \\
& \lesssim \delta h\left\|\beta_{h} \cdot \nabla_{\Gamma_{h}} v\right\|_{\mathcal{K}_{h}}^{2}+\delta^{-1} h\left(\|v\|_{\mathcal{K}_{h}}^{2}+\|v\|_{\mathcal{F}_{h}}^{2}\right)
\end{aligned}
$$

where we used the inequality Cauchy-Schwarz, the arithmetic-geometric mean inequality with parameter $\delta>0$, and Lemma 5.3.

Term II It holds

$$
\begin{aligned}
|I I| & \lesssim\left\|\alpha_{h}\right\|_{L^{\infty}\left(\mathcal{K}_{h}\right)}\|v\|_{\mathcal{K}_{h}} h\left\|\pi_{h}\left(\beta_{h} \cdot \nabla_{\Gamma_{h}} v\right)\right\|_{\mathcal{K}_{h}} \\
& \lesssim \delta^{-1} h\|v\|_{\mathcal{K}_{h}}^{2}+\delta h\left\|\pi_{h}\left(\beta_{h} \cdot \nabla_{\Gamma_{h}} v\right)\right\|_{\mathcal{K}_{h}}^{2} \\
& \lesssim \delta^{-1} h\|v\|_{\mathcal{K}_{h}}^{2}+\delta\left\|\pi_{h}\left(\beta_{h} \cdot \nabla_{\Gamma_{h}} v\right)\right\|_{\mathcal{T}_{h}}^{2} \\
& \lesssim \delta^{-1} h\|v\|_{\mathcal{K}_{h}}^{2}+\delta\left\|\beta_{h} \cdot \nabla_{\Gamma_{h}} v\right\|_{\mathcal{T}_{h}}^{2} \\
& \lesssim \delta^{-1} h\|v\|_{\mathcal{K}_{h}}^{2}+\delta h\left(\|v\|_{\mathcal{K}_{h}}^{2}+\left\|\beta_{h} \cdot \nabla_{\Gamma_{h}} v\right\|_{\mathcal{K}_{h}}^{2}+\|v\|_{\mathcal{F}_{h}}^{2}\right) \\
& \lesssim\left(\delta^{-1}+\delta\right) h\|v\|_{\mathcal{K}_{h}}^{2}+\delta h\|v\|_{\mathcal{F}_{h}}^{2}+\delta h\left\|\beta_{h} \cdot \nabla_{\Gamma_{h}} v\right\|_{\mathcal{K}_{h}}^{2}
\end{aligned}
$$

where we used Hölder's inequality, the arithmetic-geometric mean inequality with parameter $\delta>0$, the inverse estimate (4.9) to pass from $\mathcal{K}_{h}$ to $\mathcal{T}_{h}$, the boundedness (4.22) of $\pi_{h}$ on $L^{2}\left(\mathcal{T}_{h}\right)$, Lemma 5.4 , and finally we rearranged the terms.

Term III It holds

$$
\begin{aligned}
|I I I| & \lesssim h\left|\left\|v \left|\left\|\mathcal{F}_{h} \mid\right\| h \pi_{h}\left(\beta_{h} \cdot \nabla v\right) \| \mathcal{F}_{h}\right.\right.\right. \\
& \lesssim \delta^{-1} h\|\| v\left\|_{\mathcal{F}_{h}}^{2}+\delta h^{3}\right\|\left\|\pi_{h}\left(\beta_{h} \cdot \nabla v\right)\right\| \|_{\mathcal{F}_{h}}^{2} \\
& \lesssim \delta^{-1} h\|\| v\left\|_{\mathcal{F}_{h}}^{2}+\delta h^{2}\right\| \nabla \pi_{h}\left(\beta_{h} \cdot \nabla v\right) \|_{\mathcal{T}_{h}}^{2} \\
& \lesssim \delta^{-1} h\|\mid\| v\left\|_{\mathcal{F}_{h}}^{2}+\delta\right\| \pi_{h}\left(\beta_{h} \cdot \nabla v\right) \|_{\mathcal{T}_{h}}^{2} \\
& \lesssim \delta^{-1} h\|v\|\left\|_{\mathcal{F}_{h}}^{2}+\delta\right\| \beta_{h} \cdot \nabla v \|_{\mathcal{T}_{h}}^{2}
\end{aligned}
$$




$$
\begin{aligned}
& \lesssim \delta^{-1} h\|\| v \|_{\mathcal{F}_{h}}^{2}+\delta h\left(\|v\|_{\mathcal{K}_{h}}^{2}+\left\|\beta_{h} \cdot \nabla_{\Gamma_{h}} v\right\|_{\mathcal{K}_{h}}^{2}+\|\| v\|\|_{\mathcal{F}_{h}}^{2}\right) \\
& \lesssim \delta h\|v\|_{\mathcal{K}_{h}}^{2}+\left(\delta^{-1}+\delta\right) h\|\| v\left\|_{\mathcal{F}_{h}}^{2}+\delta h\right\| \beta_{h} \cdot \nabla_{\Gamma_{h}} v \|_{\mathcal{K}_{h}}^{2}
\end{aligned}
$$

where we used the Cauchy-Schwarz inequality, the arithmetic-geometric mean inequality with parameter $\delta>0$, the inverse estimate (4.8) to pass from $\mathcal{F}_{h}$ to $\mathcal{T}_{h}$, an inverse inequality to remove the gradient, the boundedness (4.22) of $\pi_{h}$ on $L^{2}\left(\mathcal{T}_{h}\right)$, Lemma 5.4, and finally we rearranged the terms.

Proposition 5.1 There is a positive constant $m_{3}$ such that

$$
m_{3}\|\mid\| v \|_{h} \leq \sup _{w \in V_{h} \backslash\{0\}} \frac{A_{h}(v, w)}{\|w\|_{h}} \quad \forall v \in V_{h}
$$

for all $h \in\left(0, h_{0}\right]$ with $h_{0}$ small enough.

Proof Setting $w=v+\gamma h \pi_{h}\left(\beta_{h} \cdot \nabla_{\Gamma_{h}} v\right)$, for some positive parameter $\gamma$, we get

$$
\begin{aligned}
A_{h}(v, w) & =A_{h}(v, v)+\gamma A_{h}\left(v, h \pi_{h}\left(\beta_{h} \cdot \nabla_{\Gamma_{h}} v\right)\right) \\
& \geq A_{h}(v, v)+\gamma m_{1} h\left\|\beta_{h} \cdot \nabla_{\Gamma_{h}} v\right\|_{\mathcal{K}_{h}}^{2}-\gamma m_{2} A_{h}(v, v) \\
& =\left(1-\gamma m_{2}\right) A_{h}(v, v)+\gamma m_{1} h\left\|\beta_{h} \cdot \nabla_{\Gamma_{h}} v\right\|_{\mathcal{K}_{h}}^{2} \\
& =\left(1-\gamma m_{2}\right) m_{0}\left(\|v\|_{\mathcal{K}_{h}}^{2}+h\left\|\left[n_{F} \cdot \nabla v\right]\right\|_{\mathcal{F}_{h}}^{2}\right)+\gamma m_{1} h\left\|\beta_{h} \cdot \nabla_{\Gamma_{h}} v\right\|_{\mathcal{K}_{h}}^{2} \\
& \geq \tilde{m}_{3}\|v\|_{h}^{2}
\end{aligned}
$$

where we used Lemmas 5.5, 5.6, and choose $0<\gamma$ small enough. Using the bound

$$
\left\|\left|w\left\|_{h} \leq C \mid\right\| v \|_{h}\right.\right.
$$

the desired estimate follows with $m_{3}=\tilde{m}_{3} / C$.

Verification of (5.90) Using the triangle inequality

$$
\left\|v+\gamma h \pi_{h}\left(\beta_{h} \cdot \nabla_{\Gamma_{h}} v\right)\right\|_{h}^{2} \lesssim\|\mid v\|_{h}^{2}+\gamma^{2}\left\|h \pi_{h}\left(\beta_{h} \cdot \nabla_{\Gamma_{h}} v\right)\right\| \|_{h}^{2}
$$

where the second term takes the form

$$
\begin{aligned}
\left\|h \pi_{h}\left(\beta_{h} \cdot \nabla_{\Gamma_{h}} v\right)\right\| \|_{h}^{2}= & h^{2}\left\|\pi_{h}\left(\beta_{h} \cdot \nabla_{\Gamma_{h}} v\right)\right\|_{\mathcal{K}_{h}}^{2} \\
& +h^{3}\left\|\beta_{h} \cdot \nabla_{\Gamma_{h}} \pi_{h}\left(\beta_{h} \cdot \nabla_{\Gamma_{h}} v\right)\right\|_{\mathcal{K}_{h}}^{2} \\
& +h^{3}\|\| \pi_{h}\left(\beta_{h} \cdot \nabla_{\Gamma_{h}} v\right) \|_{\mathcal{F}_{h}}^{2} \\
= & I+I I+I I I
\end{aligned}
$$


Term I It holds

$$
\begin{aligned}
I & =h^{2}\left\|\pi_{h}\left(\beta_{h} \cdot \nabla_{\Gamma_{h}} v\right)\right\|_{\mathcal{K}_{h}}^{2} \\
& \lesssim h\left\|\pi_{h}\left(\beta_{h} \cdot \nabla_{\Gamma_{h}} v\right)\right\|_{\mathcal{T}_{h}}^{2} \\
& \lesssim h\left\|\beta_{h} \cdot \nabla_{\Gamma_{h}} v\right\|_{\mathcal{T}_{h}}^{2} \\
& \lesssim h^{2}\|v\|_{\mathcal{K}_{h}}^{2}+h^{2}\left\|\beta_{h} \cdot \nabla_{\Gamma_{h}} v\right\|_{\mathcal{K}_{h}}^{2}+h^{2}\|v\|_{\mathcal{F}_{h}}^{2}
\end{aligned}
$$

where we used the inverse estimate (4.9) to pass from $\mathcal{K}_{h}$ to $\mathcal{T}_{h}$, the boundedness (4.22) of $\pi_{h}$ on $L^{2}\left(\mathcal{T}_{h}\right)$, and at last Lemma 5.4.

Term II It holds

$$
\begin{aligned}
h^{3}\left\|\beta_{h} \cdot \nabla_{\Gamma_{h}} \pi_{h}\left(\beta_{h} \cdot \nabla_{\Gamma_{h}} v\right)\right\|_{\mathcal{K}_{h}}^{2} & \lesssim h^{2}\left\|\beta_{h} \cdot \nabla_{\Gamma_{h}} \pi_{h}\left(\beta_{h} \cdot \nabla_{\Gamma_{h}} v\right)\right\|_{\mathcal{T}_{h}}^{2} \\
& \lesssim\left\|\pi_{h}\left(\beta_{h} \cdot \nabla_{\Gamma_{h}} v\right)\right\|_{\mathcal{T}_{h}}^{2} \\
& \lesssim\left\|\beta_{h} \cdot \nabla_{\Gamma_{h}} v\right\|_{\mathcal{T}_{h}}^{2} \\
& \lesssim h\|v\|_{\mathcal{K}_{h}}^{2}+h\left\|\beta_{h} \cdot \nabla_{\Gamma_{h}} v\right\|_{\mathcal{K}_{h}}^{2}+h\|v\| \|_{\mathcal{F}_{h}}^{2}
\end{aligned}
$$

where we used the inverse estimate (4.9) to pass from $\mathcal{K}_{h}$ to $\mathcal{T}_{h}$, an inverse estimate to remove the transport derivative, the boundedness (4.22) of $\pi_{h}$ on $L^{2}\left(\mathcal{T}_{h}\right)$, and finally we used Lemma 5.4.

Term III It holds

$$
\begin{aligned}
h^{3}\|\| \pi_{h}\left(\beta_{h} \cdot \nabla_{\Gamma_{h}} v\right) \|\left.\right|_{\mathcal{F}_{h}} ^{2} & \lesssim h^{2}\left\|\nabla \pi_{h}\left(\beta_{h} \cdot \nabla_{\Gamma_{h}} v\right)\right\|_{\mathcal{T}_{h}}^{2} \\
& \lesssim\left\|\pi_{h}\left(\beta_{h} \cdot \nabla_{\Gamma_{h}} v\right)\right\|_{\mathcal{T}_{h}}^{2} \\
& \lesssim\left\|\beta_{h} \cdot \nabla_{\Gamma_{h}} v\right\|_{\mathcal{T}_{h}}^{2} \\
& \lesssim h\|v\|_{\mathcal{K}_{h}}^{2}+h\left\|\beta_{h} \cdot \nabla_{\Gamma_{h}} v\right\|_{\mathcal{K}_{h}}^{2}+h\|\| v\|\|_{\mathcal{F}_{h}}^{2}
\end{aligned}
$$

where we used the inverse inequality (4.8) to pass from $\mathcal{F}_{h}$ to $\mathcal{T}_{h}$, an inverse estimate to remove the gradient, the boundedness (4.22) of $\pi_{h}$ on $L^{2}\left(\mathcal{T}_{h}\right)$, and finally we used Lemma 5.4 .

Conclusion of verification of (5.90) Combining the estimates of Terms I - I I I we get

$$
\left\||| h \pi_{h}\left(\beta_{h} \cdot \nabla_{\Gamma_{h}} v\right)\right\|\left\|_{h}^{2} \lesssim h\right\| v\left\|_{\mathcal{K}_{h}}^{2}+h\right\| \beta_{h} \cdot \nabla_{\Gamma_{h}} v\left\|_{\mathcal{K}_{h}}^{2}+h\right\|\|v\|\left\|_{\mathcal{F}_{h}}^{2} \lesssim\right\|\|v\| \|_{h}^{2}
$$

and therefore, in view of (5.91), we conclude that (5.90) holds.

Proposition 5.2 It holds

$$
h^{3 / 4}\left\|\nabla_{\Gamma_{h}} v\right\|_{\mathcal{K}_{h}} \lesssim\|\| v \|_{h} \quad \forall v \in V_{h}
$$

for all $h \in\left(0, h_{0}\right]$ with $h_{0}$ small enough. 
Proof Using partial integration followed by Cauchy-Schwarz we have

$$
\begin{aligned}
\left\|\nabla_{\Gamma_{h}} v\right\|_{\mathcal{K}_{h}}^{2} & =\left(\nabla_{\Gamma_{h}} v, \nabla_{\Gamma_{h}} v\right)_{\mathcal{K}_{h}} \\
& =-\left(v,\left[n_{E} \cdot \nabla v\right]\right)_{\mathcal{E}_{h}} \\
& \leq \underbrace{\|v\|_{\mathcal{E}_{h}}}_{I} \underbrace{\left\|\left[n_{E} \cdot \nabla v\right]\right\|_{\mathcal{E}_{h}}}_{I I}
\end{aligned}
$$

Term I Using Lemma 5.2 we directly obtain

$$
\|v\|_{\mathcal{E}_{h}}^{2} \lesssim h^{-1}\left(\|v\|_{\mathcal{K}_{h}}^{2}+h^{2}\|\| v \|\left.\right|_{\mathcal{F}_{h}} ^{2}\right) \lesssim h^{-1}\|v\|_{\mathcal{K}_{h}}^{2}+h\|\| v\|\|_{\mathcal{F}_{h}}^{2}
$$

and we conclude that

$$
h\|v\|_{\mathcal{E}_{h}}^{2} \lesssim\|v\|_{\mathcal{K}_{h}}^{2}+h^{2}\|\| v\left\|\left.\right|_{\mathcal{F}_{h}} ^{2} \lesssim\right\|\|v\| \|_{h}^{2}
$$

Term II We have the estimates

$$
\begin{aligned}
\left\|\left[n_{E} \cdot \nabla v\right]\right\|_{\mathcal{E}_{h}}^{2} & \lesssim h^{-1}\left\|\left[n_{E} \cdot \nabla v\right]\right\|_{\mathcal{F}_{h}}^{2} \\
& \lesssim h^{-1}\left\|\left[n_{E}\right] \cdot\langle\nabla v\rangle\right\|_{\mathcal{F}_{h}}^{2}+h^{-1}\left\|\left\langle n_{E}\right\rangle \cdot[\nabla v]\right\|_{\mathcal{F}_{h}}^{2} \\
& \lesssim h^{-1}\|h \nabla v\|_{\mathcal{F}_{h}}^{2}+h^{-1}\left\|\left\langle n_{E}\right\rangle \cdot n_{F}\left[n_{F} \cdot \nabla v\right]\right\|_{\mathcal{F}_{h}}^{2} \\
& \lesssim h^{-2}\|h \nabla v\|_{\mathcal{T}_{h}}^{2}+h^{-1}\left\|\left[n_{F} \cdot \nabla v\right]\right\|_{\mathcal{F}_{h}}^{2} \\
& \lesssim h^{-2}\|v\|_{\mathcal{T}_{h}}^{2}+h^{-1}\left\|\left[n_{F} \cdot \nabla v\right]\right\|_{\mathcal{F}_{h}}^{2} \\
& \lesssim h^{-2}\left(h\|v\|_{\mathcal{K}_{h}}^{2}+h\left\|\left[n_{F} \cdot \nabla v\right]\right\|_{\mathcal{F}_{h}}^{2}\right)+h^{-1}\left\|\left[n_{F} \cdot \nabla v\right]\right\|_{\mathcal{F}_{h}}^{2} \\
& \lesssim h^{-1}\left(\|v\|_{\mathcal{K}_{h}}^{2}+\left\|\left[n_{F} \cdot \nabla v\right]\right\|_{\mathcal{F}_{h}}^{2}\right)
\end{aligned}
$$

Here we used the inverse inequality (4.7) to pass from $\mathcal{E}_{h}$ to $\mathcal{F}_{h}$, the identity $[a b]=$ $[a]\langle b\rangle+\langle a\rangle[b]$, where $\langle a\rangle=\left(a^{+}+a^{-}\right) / 2$ is the average of a discontinuous function, the fact that the tangent gradient is continuous at a face, the inverse inequality (4.8) to pass from $\mathcal{F}_{h}$ to $\mathcal{T}_{h}$ in the first term and a direct estimate for the second, Lemma 5.1 for the first term, and finally we collected the terms. We conclude that

$$
h^{2}\left\|\left[n_{E} \cdot \nabla v\right]\right\|_{\mathcal{E}_{h}}^{2} \lesssim\|v\| \|_{h}^{2}
$$

Conclusion Combining (5.110) with the estimates (5.112) and (5.120) we obtain

$$
h^{3}\left\|\nabla_{\Gamma_{h}} v\right\|_{\mathcal{K}_{h}}^{4} \lesssim h\|v\|_{\mathcal{E}_{h}}^{2} h^{2}\left\|\left[n_{E} \cdot \nabla_{\Gamma_{h}} v\right]\right\|_{\mathcal{E}_{h}}^{2} \lesssim\|\| v\|\|_{h}^{4}
$$

which concludes the proof. 


\section{Error estimates}

\subsection{Assumptions on the coefficients for the error estimates}

In addition to the assumptions on the coefficients used in the stability estimates, see Sect. 5.2, we here formulate further assumptions needed in the error analysis. In Sect. 6.4 we verify these assumptions.

Assumption Let $\beta_{h}, \alpha_{h}$, and $f_{h}$, be elementwise linear polynomial approximations of $|B| B^{-1} \beta,|B| \alpha$ and $|B| f$. Assume that there are constants such that

$$
\begin{aligned}
\left\||B| B^{-1} \beta-\beta_{h}\right\|_{L^{\infty}\left(\mathcal{K}_{h}\right)} & \leq C_{\beta} h^{2}, \\
\left\||B| \alpha-\alpha_{h}\right\|_{\infty}\left(\mathcal{K}_{h}\right) & \leq C_{\alpha} h^{2}, \\
\left\||B| f-f_{h}\right\|_{L^{\infty}\left(\mathcal{K}_{h}\right)} & \leq C_{f} h^{2}
\end{aligned}
$$

\subsection{Strang's Lemma}

Define the forms

$$
a(v, w)=\left(\beta \cdot \nabla_{\Gamma} v, w\right)_{\Gamma}+(\alpha v, w)_{\Gamma}, \quad l(v)=(f, v)_{\Gamma}
$$

Then the exact solution $u$ to the convection problem (2.3), see Proposition 2.1, satisfies

$$
a(u, v)=l(v) \quad \forall v \in L^{2}(\Gamma)
$$

We then have the following Strang Lemma.

Lemma 6.1 Let $u$ be the solution to (2.3), $u_{h}$ the finite element approximation defined by (3.6), then the following estimate holds

$$
\begin{aligned}
\left\|u^{e}-u_{h}\right\|_{h} \lesssim & h^{3 / 2}\|u\|_{H^{2}(\Gamma)} \\
& +\sup _{v \in V_{h} \backslash 0} \frac{a\left(\left(\pi_{h} u^{e}\right)^{l}, v^{l}\right)-a_{h}\left(\pi_{h} u^{e}, v\right)}{\|v\|_{h}} \\
& +\sup _{v \in V_{h} \backslash 0} \frac{l\left(v^{l}\right)-l_{h}(v)}{\left.\left\|v^{l}\right\|\right|_{h}}
\end{aligned}
$$

Remark 6.1 We return to the construction of $\beta_{h}$ that satisfies (6.2) in the following section. In fact, we will see that we need a stronger assumption on $\beta_{h}$ in order to guarantee optimal order convergence.

Proof Adding and subtracting an interpolant $\pi_{h} u^{e}$, and then using the triangle inequality we obtain

$$
\left\|\left|u^{e}-u_{h}\right|\right\|_{h} \leq\|\| u-\pi_{h} u^{e}\left|\left\|_{h}+\right\|\right| \pi_{h} u^{e}-u_{h} \|_{h}
$$




$$
\lesssim h^{3 / 2}\|u\|_{H^{2}(\Gamma)}+\|\| \pi_{h} u^{e}-u_{h} \|_{h}
$$

where we used the interpolation estimate (4.26) to estimate the first term. Proceeding with the second term we employ the inf-sup estimate in Proposition 5.1 to get the bound

$$
\left\|\pi_{h} u^{e}-u_{h}\right\| \|_{h} \lesssim \sup _{v \in V_{h} \backslash\{0\}} \frac{A_{h}\left(\pi_{h} u-u_{h}, v\right)}{\|v\|_{h}}
$$

Adding and subtracting the exact solution, and using Galerkin orthogonality (3.6) the numerator may be written in the following form

$$
\begin{aligned}
A_{h}\left(\pi_{h} u^{e}-u_{h}, v\right)= & A_{h}\left(\pi_{h} u^{e}, v\right)-l_{h}(v) \\
= & A_{h}\left(\pi_{h} u^{e}, v\right)-a\left(\left(\pi_{h} u^{e}\right)^{l}, v^{l}\right) \\
& +a\left(\left(\pi_{h} u^{e}\right)^{l}-u, v^{l}\right)+l\left(v^{l}\right)-l_{h}(v) \\
= & \underbrace{a_{h}\left(\pi_{h} u^{e}, v\right)-a\left(\left(\pi_{h} u^{e}\right)^{l}, v^{l}\right)}_{I}+\underbrace{j_{h}\left(\pi_{h} u^{e}, v\right)}_{I I} \\
& +\underbrace{a\left(\left(\pi_{h} u^{e}\right)^{l}-u, v^{l}\right)}_{I I I}+\underbrace{l\left(v^{l}\right)-l_{h}(v)}_{I V} \\
= & I+I I+I I I+I V
\end{aligned}
$$

Here terms $I$ and $I V$ gives rise to the second and third terms on the right hand side in (6.6) and II and III can be estimated as follows

$$
|I I|+|I I I| \lesssim h^{3 / 2}\|u\|_{H^{2}(\Gamma)} \mid\|v\| \|_{h}
$$

which together with (6.9) yields (6.6). It remains to verify (6.14).

Term II This term is immediately estimated using (4.27) as follows

$$
\begin{aligned}
|I I| & =\left|j_{h}\left(\pi_{h} u^{e}-u^{e}, v\right)\right| \\
& \lesssim h\left\|\left|\pi_{h} u^{e}-u^{e}\right|\right\| \mathcal{F}_{h}\left|\|v \mid\| \mathcal{F}_{h}\right. \\
& \lesssim h^{3 / 2}\|u\|_{H^{2}(\Gamma)} \mid\|v\| \|_{h}
\end{aligned}
$$

Term III Using Green's formula and changing domain of integration from $\Gamma$ to $\Gamma_{h}$ we obtain

$$
\begin{aligned}
a\left(\left(\pi_{h} u^{e}\right)^{l}-u, v^{l}\right)= & \left(\beta \cdot \nabla_{\Gamma}\left(\left(\pi_{h} u^{e}\right)^{l}-u\right), v^{l}\right)_{\Gamma} \\
& +\left(\alpha\left(\left(\pi_{h} u^{e}\right)^{l}-u\right), v^{l}\right)_{\Gamma} \\
= & -\left(\left(\pi_{h} u^{e}\right)^{l}-u, \beta \cdot \nabla_{\Gamma} v^{l}\right)_{\Gamma} \\
& +\left(\left(\alpha-\operatorname{div}_{\Gamma} \beta\right)\left(\left(\pi_{h} u^{e}\right)^{l}-u\right), v^{l}\right)_{\Gamma} \\
= & I I I_{1}+I I I_{2}
\end{aligned}
$$


Changing domain of integration from $\Gamma$ to $\Gamma_{h}$ and using the identity (4.15) we obtain

$$
\begin{aligned}
I I I_{1}= & -\left(\left(\pi_{h} u^{e}\right)^{l}-u, \beta \cdot \nabla_{\Gamma} v^{l}\right)_{\Gamma} \\
= & -\left(\pi_{h} u^{e}-u^{e},|B| \beta \cdot B^{-T} \nabla_{\Gamma_{h}} v\right)_{\Gamma_{h}} \\
= & -\left(\pi_{h} u^{e}-u^{e},|B| B^{-1} \beta \cdot \nabla_{\Gamma_{h}} v\right)_{\Gamma_{h}} \\
= & -\left(\pi_{h} u^{e}-u^{e}, \beta_{h} \cdot \nabla_{\Gamma_{h}} v\right)_{\Gamma_{h}} \\
& \quad-\left(\pi_{h} u^{e}-u^{e},\left(|B| B^{-1} \beta-\beta_{h}\right) \cdot \nabla_{\Gamma_{h}} v\right)_{\Gamma_{h}} \\
\leq & \left\|\pi_{h} u^{e}-u^{e}\right\|_{\Gamma_{h}}\left\|\beta_{h} \cdot \nabla_{\Gamma_{h}} v\right\|_{\Gamma_{h}} \\
& \quad+\left\|\pi_{h} u^{e}-u^{e}\right\|_{\Gamma_{h}}\left\||B| B^{-1} \beta-\beta_{h}\right\|_{L^{\infty}\left(\Gamma_{h}\right)}\left\|\nabla_{\Gamma_{h}} v\right\|_{\Gamma_{h}} \\
= & \star
\end{aligned}
$$

where we added and subtracted $\beta_{h}$ and used the Cauchy-Schwarz inequality. Next using an inverse estimate followed by Lemma 5.1 we obtain

$$
\left\|\nabla_{\Gamma_{h}} v\right\|_{\mathcal{K}_{h}}^{2} \lesssim h^{-1}\|\nabla v\|_{\mathcal{T}_{h}}^{2} \lesssim h^{-3}\|v\|_{\mathcal{T}_{h}}^{2} \lesssim h^{-3}\left(h\|v\|_{\mathcal{K}_{h}}^{2}+h\|\| v \|_{\mathcal{F}_{h}}^{2}\right) \lesssim h^{-3}\|v\| \|_{h}^{2}
$$

and using the interpolation estimate (4.25) and assumption (6.1) we have

$$
\begin{aligned}
& \star \lesssim h^{-1 / 2} \underbrace{\left\|\pi_{h} u^{e}-u^{e}\right\|_{\Gamma_{h}}}_{\lesssim h^{2}\|u\|_{H^{2}(\Gamma)}} \underbrace{h^{1 / 2}\left\|\beta_{h} \cdot \nabla_{\Gamma_{h}} v\right\|_{\Gamma_{h}}}_{\lesssim\|v\|_{h}} \\
& +\underbrace{\left\|\pi_{h} u^{e}-u^{e}\right\|_{\Gamma_{h}}}_{\lesssim h^{2}\|u\|_{H^{2}(\Gamma)}} \underbrace{\left\||B| B^{-1} \beta-\beta_{h}\right\|_{L^{\infty}\left(\Gamma_{h}\right)}}_{\lesssim C_{\beta} h^{2}} h^{-3 / 2}\|v\|_{h} \\
& \lesssim\left(h^{3 / 2}+C_{\beta} h^{5 / 2}\right)\|u\|_{H^{2}(\Gamma)}|\|v\||_{h}
\end{aligned}
$$

To estimate $\mathrm{II}_{2}$ we change domain of integration from $\Gamma$ to $\Gamma_{h}$ and use the interpolation estimate

$$
\begin{aligned}
I I I_{2} & =\left(\left(\alpha-\operatorname{div}_{\Gamma} \beta\right)^{e}|B|\left(\pi_{h} u^{e}-u^{e}\right), v\right)_{\Gamma_{h}} \\
& \lesssim\left\|\alpha-\operatorname{div}_{\Gamma} \beta\right\|_{L^{\infty}(\Gamma)}\left\|\pi_{h} u^{e}-u^{e}\right\|_{\Gamma_{h}}\|v\|_{\Gamma_{h}} \\
& \lesssim h^{2}\|u\|_{H^{2}(\Gamma)}\|v\|_{\Gamma_{h}}
\end{aligned}
$$

Together the bounds (6.29) and (6.32) give the desired estimate

$$
|I I I| \lesssim h^{3 / 2}\|u\|_{H^{2}(\Gamma)} \mid\|v\|_{h}
$$




\subsection{Quadrature error estimates}

\section{Lemma 6.2 If (6.1-6.3) hold then}

$$
\begin{aligned}
\left|a\left(\left(\pi_{h} u^{e}\right)^{l}, v^{l}\right)-a_{h}\left(\left(\pi_{h} u^{e}\right), v\right)\right| & \lesssim\left(C_{\beta}+C_{\alpha}\right) h^{2}\|u\|_{H^{1}(\Gamma)} \mid\|w\|_{h} \quad \forall v \in V_{h} \\
\left|l\left(v^{l}\right)-l_{h}(v)\right| & \lesssim C_{f} h^{2}\|v\|_{\Gamma_{h}} \quad \forall v \in V_{h}
\end{aligned}
$$

Proof Changing domain of integration to $\Gamma_{h}$ and using the identity (4.15) for the tangential derivative of a lifted function we obtain

$$
\begin{aligned}
(\beta \cdot & \left.\nabla_{\Gamma}\left(\pi_{h} u^{e}\right)^{l}, w^{l}\right)_{\mathcal{K}_{h}^{l}}-\left(\beta_{h} \cdot \nabla_{\Gamma_{h}}\left(\pi_{h} u^{e}\right), w\right)_{\mathcal{K}_{h}} \\
& =\left(|B|\left(\beta \cdot \nabla_{\Gamma}\left(\pi_{h} u^{e}\right)^{l}\right)^{e}-\beta_{h} \cdot\left(\nabla_{\Gamma_{h}}\left(\pi_{h} u^{e}\right)\right), w\right) \mathcal{K}_{h} \\
& =\left(|B|\left(\beta \cdot B^{-T} \nabla_{\Gamma_{h}}\left(\pi_{h} u^{e}\right)\right)^{e}-\beta_{h} \cdot \nabla_{\Gamma_{h}}\left(\pi_{h} u^{e}\right), w\right)_{\mathcal{K}_{h}} \\
& =\left(\left(|B| B^{-1} \beta-\beta_{h}\right) \cdot \nabla_{\Gamma_{h}}\left(\pi_{h} u^{e}\right), w\right) \mathcal{K}_{h} \\
& =\left\||B| B^{-1} \beta-\beta_{h}\right\|_{L^{\infty}\left(\Gamma_{h}\right)}\left\|\nabla_{\Gamma_{h}}\left(\pi_{h} u^{e}\right)\right\|_{\mathcal{K}_{h}}\|w\|_{\mathcal{K}_{h}} \\
& =\left\||B| B^{-1} \beta-\beta_{h}\right\|_{L^{\infty}\left(\Gamma_{h}\right)}\|u\|_{H^{1}(\Gamma)}\|w\|_{\mathcal{K}_{h}}
\end{aligned}
$$

where at last we used the $H^{1}$ stability of the interpolant to conclude that

$$
\left\|\nabla_{\Gamma_{h}}\left(\pi_{h} u^{e}\right)\right\|_{\mathcal{K}_{h}}^{2} \lesssim h^{-1}\left\|\nabla_{\Gamma_{h}}\left(\pi_{h} u^{e}\right)\right\|_{\mathcal{T}_{h}}^{2} \lesssim h^{-1}\left\|\nabla u^{e}\right\|_{\mathcal{T}_{h}}^{2} \lesssim\|u\|_{H^{1}(\Gamma)}^{2}
$$

Using the same approach and (6.2) we have

$$
\begin{aligned}
\left|\left(\alpha\left(\pi_{h} u^{e}\right)^{l}, v^{l}\right)_{\mathcal{K}_{h}^{l}}-\left(\alpha_{h}\left(\pi_{h} u^{e}\right), v\right)_{\mathcal{K}_{h}}\right| & =\left|\left(\left(|B| \alpha^{e}-\alpha_{h}\right)\left(\pi_{h} u^{e}\right), v\right)_{\mathcal{K}_{h}}\right| \\
& \leq\left\||B| \alpha^{e}-\alpha_{h}\right\|_{L^{\infty}\left(\Gamma_{h}\right)}\left\|\pi_{h} u^{e}\right\| \mathcal{K}_{h}\|v\|_{\mathcal{K}_{h}} \\
& \lesssim\left\||B| \alpha^{e}-\alpha_{h}\right\|_{L^{\infty}\left(\Gamma_{h}\right)}\|u\|_{\Gamma}\|v\|_{\mathcal{K}_{h}} \\
& \lesssim h^{2}\|u\|_{\Gamma}\|v\|_{h}
\end{aligned}
$$

which together with (6.40) yield (6.34).

Finally, (6.35) follows using (6.3),

$$
\begin{aligned}
\left|\left(f, v^{l}\right)_{\mathcal{K}_{h}^{l}}-\left(f_{h}, v^{l}\right)_{\mathcal{K}_{h}}\right| & =\left|\left(|B| f^{e}-f_{h}, v\right)_{\mathcal{K}_{h}}\right| \\
& \leq\left\||B| f^{e}-f_{h}\right\|_{L^{\infty}\left(\Gamma_{h}\right)}\|v\|_{\mathcal{K}_{h}} \\
& \lesssim h^{2}\|v\|_{\mathcal{K}_{h}}
\end{aligned}
$$

Remark 6.2 Note that the fact that $\pi_{h} u^{e}$ and $\left(\pi_{h} u^{e}\right)^{l}$ appear in the first slots in the bilinear forms in (6.34) is crucial since we get $L^{2}$ control over the full tangent gradient $\nabla_{\Gamma_{h}}\left(\pi_{h} u^{e}\right)$, i.e. $\left\|\nabla_{\Gamma_{h}}\left(\pi_{h} u^{e}\right)\right\|_{\Gamma_{h}} \lesssim\|u\|_{H^{1}(\Gamma)}$, using stability of the interpolant, while 
the corresponding control of the discrete solution $u_{h}$ provided by Proposition 5.2 is only $h^{3 / 4}\left\|\nabla_{\Gamma_{h}} u_{h}\right\|_{\mathcal{K}_{h}} \lesssim\|\| u_{h}\left\|_{h} \lesssim \sup _{v \in V_{h}} \frac{A_{h}\left(u_{h}, v\right)}{\|\mid\| v\|\|_{h}} \lesssim\right\| f_{h} \|_{\mathcal{K}_{h}}$, indicating a higher demand on the accuracy of $\beta_{h}$ in order to achieve optimal order of convergence.

Remark 6.3 Note that we do not have to take $|B|$ into account in (6.1-6.3) since ||$B|-1| \sim h^{2}$. Thus we could instead use the simplified assumptions

$$
\left\|B^{-1} \beta-\beta_{h}\right\|_{L^{\infty}\left(\mathcal{K}_{h}\right)} \lesssim h^{2}, \quad\left\|\alpha-\alpha_{h}\right\|_{\infty}\left(\mathcal{K}_{h}\right) \lesssim h^{2}, \quad\left\|f-f_{h}\right\|_{L^{\infty}\left(\mathcal{K}_{h}\right)} \lesssim h^{2}
$$

Furthermore, $B^{-1}=P_{\Gamma_{h}} P_{\Gamma}+O\left(h^{2}\right)$, see (4.12) in [17], and thus we may simplify the assumption on $\beta_{h}$ even further and assume that

$$
\left\|P_{\Gamma_{h}} \beta^{e}-\beta_{h}\right\|_{L^{\infty}\left(\mathcal{K}_{h}\right)} \lesssim h^{2}
$$

Remark 6.4 The mapping $\beta \mapsto|B| B^{-1} \beta$ is in fact a Piola mapping which maps tangent vectors on $\Gamma$ onto tangent vectors on $\Gamma_{h}$.

\subsection{Construction of the discrete coefficients}

Here we provide a concrete construction of coefficients that satisfy our assumptions. In order to handle the fact that a surface element $K \in \mathcal{K}_{h}$ can be arbitrarily small and is not in general shape regular we interpolate the coefficients over a larger shape regular triangle $\widetilde{K}$. More precisely, given $K \in \mathcal{K}_{h}$ we may construct a shape regular triangle $\widetilde{K}$ in the hyperplane defined by $K$ such that $K \subset \widetilde{K}$ and $\operatorname{diam}(\widetilde{K}) \lesssim h$. We can then define

$$
\widetilde{\beta}_{h}=\widetilde{I}_{h} P_{K} \beta^{e} \in P_{1}(\widetilde{K}),\left.\quad \beta_{h}\right|_{K}=\left.\widetilde{\beta}_{h}\right|_{K}
$$

where $P_{K}$ is the tangent projection associated with $K$ and $\widetilde{I}_{h}$ is the linear Lagrange interpolant associated with the triangle $\widetilde{K}$. Assuming that $\beta \in C^{2}(\Gamma)$ we have

$$
\left\|P_{K} \beta^{e}-\beta_{h}\right\|_{W_{\infty}^{m}(K)} \lesssim\left\|\left(I-\tilde{I}_{h}\right) P_{K} \beta^{e}\right\|_{W_{\infty}^{m}(\widetilde{K})} \lesssim h^{2-m}\|\beta\|_{W_{\infty}^{2}\left(\widetilde{K}^{l}\right)}, \quad m=0,1
$$

since $P_{K}$ is constant. Similar constructions can be done for $\alpha_{h}$ and $f_{h}$.

Lemma 6.3 Assuming that $\beta, \alpha, f$ are all in $C^{2}(\Gamma), \beta_{h}, \alpha_{h}$, and $f_{h}$ satisfy all the assumptions (5.31), (5.30-5.32), needed for the stability analysis, and the assumptions (6.1-6.3) needed in the quadrature error estimates, for all $h \in\left(0, h_{0}\right]$ with $h_{0}$ small enough.

Proof (5.30). Adding and subtracting $\left(\alpha-\frac{1}{2} \operatorname{div}_{\Gamma} \beta\right)^{e}$ we obtain

$$
\begin{aligned}
\alpha_{h}-\frac{1}{2} \operatorname{div}_{\Gamma_{h}} \beta_{h}= & \left(\alpha-\frac{1}{2} \operatorname{div}_{\Gamma} \beta\right)^{e} \\
& +\alpha_{h}-\alpha^{e}-\frac{1}{2}\left(\operatorname{div}_{\Gamma_{h}} \beta_{h}-\left(\operatorname{div}_{\Gamma} \beta\right)^{e}\right) \\
\geq & \alpha-\frac{1}{2} \operatorname{div}_{\Gamma} \beta
\end{aligned}
$$




$$
\begin{aligned}
& \quad-\left\|\alpha_{h}-\alpha^{e}\right\|_{L^{\infty}\left(\Gamma_{h}\right)}-\frac{1}{2}\left\|\operatorname{div}_{\Gamma_{h}} \beta_{h}-\left(\operatorname{div}_{\Gamma} \beta\right)^{e}\right\|_{L^{\infty}\left(\Gamma_{h}\right)} \\
& \gtrsim C_{1}-C_{2} h
\end{aligned}
$$

where we used (2.4) and the bounds

$$
\left\|\alpha_{h}-\alpha^{e}\right\|_{L^{\infty}\left(\Gamma_{h}\right)} \lesssim h^{2}, \quad\left\|\operatorname{div}_{\Gamma_{h}} \beta_{h}-\left(\operatorname{div}_{\Gamma} \beta\right)^{e}\right\|_{L^{\infty}\left(\Gamma_{h}\right)} \lesssim h
$$

To verify the second bound in (6.56) we first note that

$$
\operatorname{div}_{\Gamma_{h}} \beta^{e}=\operatorname{div}_{\Gamma_{h}}\left(P_{\Gamma_{h}} \beta^{e}+n_{h}\left(n_{h} \cdot \beta^{e}\right)\right)=\operatorname{div}_{\Gamma_{h}}\left(P_{\Gamma_{h}} \beta^{e}\right)
$$

which holds since $\operatorname{div}_{\Gamma_{h}}\left(n_{h}\left(n_{h} \cdot \beta^{e}\right)\right)=\left(n_{h} \cdot \beta^{e}\right) \operatorname{tr}\left(n_{h} \otimes \nabla_{\Gamma_{h}}\right)+n_{h} \cdot \nabla_{\Gamma_{h}}\left(n_{h} \cdot \beta^{e}\right)=0$ and we used the facts that $n_{h}$ is piecewise constant and orthogonal to $\nabla_{\Gamma_{h}}\left(n_{h} \cdot \beta^{e}\right)$. Next subtracting $\operatorname{div}_{\Gamma_{h}}\left(P_{\Gamma_{h}} \beta^{e}\right)$ and adding $\operatorname{div}_{\Gamma_{h}} \beta^{e}$ and using the triangle inequality we obtain

$$
\begin{aligned}
\left\|\operatorname{div}_{\Gamma_{h}} \beta_{h}-\left(\operatorname{div}_{\Gamma} \beta\right)^{e}\right\|_{L^{\infty}\left(\Gamma_{h}\right)=} & \left\|\operatorname{div}_{\Gamma_{h}} \beta_{h}-\operatorname{div}_{\Gamma_{h}}\left(P_{\Gamma_{h}} \beta^{e}\right)\right\|_{L^{\infty}\left(\Gamma_{h}\right)} \\
& +\left\|\operatorname{div}_{\Gamma_{h}} \beta^{e}-\left(\operatorname{div}_{\Gamma} \beta\right)^{e}\right\|_{L^{\infty}\left(\Gamma_{h}\right)} \\
= & I+I I
\end{aligned}
$$

Term I For $K \in \mathcal{K}_{h}$,

$$
\begin{aligned}
\left\|\operatorname{div}_{\Gamma_{h}} \beta_{h}-\operatorname{div}_{\Gamma_{h}} P_{\Gamma_{h}} \beta^{e}\right\|_{L^{\infty}(K)} & =\left\|\operatorname{div}_{\Gamma_{h}}\left(\left(\widetilde{I}_{h}-I\right) P_{\Gamma_{h}} \beta^{e}\right)\right\|_{L^{\infty}(K)} \\
& \lesssim\left\|\left(\left(\widetilde{I}_{h}-I\right) P_{\Gamma_{h}} \beta^{e}\right) \otimes \nabla_{\Gamma_{h}}\right\|_{L^{\infty}(K)} \\
& \lesssim h\|\beta\|_{W_{\infty}^{2}\left(\widetilde{K}^{l}\right)}
\end{aligned}
$$

where we used (6.52).

Term II Using the definition of the surface divergence operators we have

$$
\begin{aligned}
\left\|\operatorname{div}_{\Gamma_{h}} \beta^{e}-\left(\operatorname{div}_{\Gamma} \beta\right)^{e}\right\|_{L^{\infty}\left(\Gamma_{h}\right)} & =\left\|\operatorname{tr}\left(\beta^{e} \otimes \nabla_{\Gamma_{h}}\right)-\left(\operatorname{tr}\left(\beta \otimes \nabla_{\Gamma}\right)\right)^{e}\right\|_{L^{\infty}\left(\Gamma_{h}\right)} \\
& =\left\|\operatorname{tr}\left(\beta \otimes \nabla_{\Gamma} B\right)-\operatorname{tr}\left(\beta \otimes \nabla_{\Gamma}\right)^{e}\right\|_{L^{\infty}\left(\Gamma_{h}\right)} \\
& =\left\|\operatorname{tr}\left(B^{T} \nabla_{\Gamma} \otimes \beta\right)-\operatorname{tr}\left(\nabla_{\Gamma} \otimes \beta\right)^{e}\right\|_{L^{\infty}\left(\Gamma_{h}\right)} \\
& \lesssim\left\|B^{T} \nabla_{\Gamma} \otimes \beta-\left(\nabla_{\Gamma} \otimes \beta\right)^{e}\right\|_{L^{\infty}\left(\Gamma_{h}\right)} \\
& =\left\|B^{T} \nabla_{\Gamma} \otimes \beta-P_{\Gamma} \nabla_{\Gamma} \otimes \beta\right\|_{L^{\infty}\left(\Gamma_{h}\right)} \\
& =\left\|\left(B^{T}-P_{\Gamma}\right) \nabla_{\Gamma} \otimes \beta\right\|_{L^{\infty}\left(\Gamma_{h}\right)} \\
& \lesssim h\|\beta\|_{W_{\infty}^{1}(\Gamma)}
\end{aligned}
$$

Here, for $x \in \Gamma_{h}$, we interpret $P_{\Gamma}$ as an operator

$$
P_{\Gamma}: T_{p(x)}\left(\mathbb{R}^{3}\right) \ni \xi \mapsto\left(\left.P_{\Gamma}\right|_{p(x)} \xi\right)^{e} \in T_{x}\left(\mathbb{R}^{3}\right)
$$


i.e. the operator that first projects onto the tangent plane $T_{p(x)}(\Gamma)$ and then translates the tangent vector to a vector at $x$. We also recall that the transpose of $B$ is an operator $B^{T}: T_{p(x)}(\Gamma) \ni \xi \mapsto B^{T} \xi \in T_{x}\left(\Gamma_{h}\right) \subset T_{x}\left(\mathbb{R}^{3}\right)$. Thus we can write

$$
\left(\beta \otimes \nabla_{\Gamma}\right)^{e}=\beta \otimes \nabla_{\Gamma} P_{\Gamma}
$$

We then have the identities

$$
\begin{aligned}
B^{T}-P_{\Gamma} & =P_{\Gamma_{h}}(I-\rho \mathcal{H}) P_{\Gamma}-P_{\Gamma} \\
& =\left(P_{\Gamma_{h}}-P_{\Gamma}\right) P_{\Gamma}-\rho P_{\Gamma_{h}} \mathcal{H} P_{\Gamma} \\
& =\left(Q_{\Gamma}-Q_{\Gamma_{h}}\right) P_{\Gamma}-\rho P_{\Gamma_{h}} \mathcal{H} P_{\Gamma} \\
& =-n_{h} \otimes\left(P_{\Gamma}\left(n_{h}-n_{h}\right)\right)-\rho P_{\Gamma_{h}} \mathcal{H} P_{\Gamma}
\end{aligned}
$$

where $Q_{\Gamma}=I-P_{\Gamma}$ and $Q_{\Gamma_{h}}=I-P_{\Gamma_{h}}$. We thus have the estimate

$$
\begin{aligned}
\left\|B^{T}-P_{\Gamma}\right\|_{L^{\infty}\left(\Gamma_{h}\right)} & \leq\left\|n_{h} \otimes\left(P_{\Gamma}\left(n_{h}-n\right)\right)\right\|_{L^{\infty}\left(\Gamma_{h}\right)}+\left\|\rho P_{\Gamma_{h}} \mathcal{H} P_{\Gamma}\right\|_{L^{\infty}\left(\Gamma_{h}\right)} \\
& \leq\left\|n_{h}-n\right\|_{L^{\infty}\left(\Gamma_{h}\right)}+\|\rho\|_{L^{\infty}\left(\Gamma_{h}\right)}\|\mathcal{H}\|_{L^{\infty}\left(\Gamma_{h}\right)} \\
& \lesssim h+h^{2}
\end{aligned}
$$

where we at last used (3.1) and (4.12).

(5.31). Adding and subtracting $P_{\Gamma_{h}} \beta^{e}$ we have

$$
\begin{aligned}
\left\|\beta_{h}-\beta_{h, x}\right\|_{L^{\infty}\left(\mathcal{K}_{h, x}\right) \leq} & \left\|\beta_{h}-P_{\Gamma_{h}} \beta^{e}\right\|_{L^{\infty}\left(\mathcal{K}_{h, x}\right)}+\left\|P_{\Gamma_{h}} \beta^{e}-\beta_{h, x}\right\|_{L^{\infty}\left(\mathcal{K}_{h, x}\right)} \\
\leq & \left\|\beta_{h}-P_{\Gamma_{h}} \beta^{e}\right\|_{L^{\infty}\left(\mathcal{K}_{h, x}\right)}+\left\|\left(P_{\Gamma_{h}}-P_{\Gamma}\right) \beta^{e}\right\|_{L^{\infty}\left(\mathcal{K}_{h, x}\right)} \\
& \quad+\left\|\beta^{e}-\beta_{h, x}\right\|_{L^{\infty}\left(\mathcal{K}_{h, x}\right)} \\
\lesssim & h^{2}+h+h
\end{aligned}
$$

where we used (6.52) to estimate the first term on the right hand side of (6.80) and for the second term we used the fact that $\beta=P_{\Gamma} \beta$ since $\beta$ is tangential, and the third term is estimated using Taylor's formula.

(5.32). Splitting the jump by adding and subtracting $\beta$ we get

$$
\begin{aligned}
\left\|\left[n_{E} \cdot \beta_{h}\right]\right\|_{L^{\infty}(E) \leq} \leq & \left\|\left(n_{E} \cdot\left(\beta_{h}-\beta^{e}\right)\right)^{+}\right\|_{L^{\infty}(E)} \\
& +\left\|\left(n_{E} \cdot\left(\beta_{h}-\beta^{e}\right)\right)^{-}\right\|_{L^{\infty}(E)}+\left\|\left[n_{E}\right] \cdot \beta^{e}\right\|_{L^{\infty}(E)} \\
= & I+I I+I I I
\end{aligned}
$$

Terms $I$ and II We consider $I$ since these terms are of the same form. Using the fact that $n_{E}^{+}$is a tangent vector to $K$ and definition of $\beta_{h}$ we obtain

$$
\begin{aligned}
\left\|n_{E}^{+} \cdot\left(\beta_{h}^{+}-\beta^{e}\right)\right\|_{L^{\infty}(E)} & =\left\|n_{E}^{+} \cdot\left(\beta_{h}^{+}-P_{K^{+}} \beta^{e}\right)\right\|_{L^{\infty}(E)} \\
& \leq\left\|\beta_{h}^{+}-P_{K^{+}} \beta^{e}\right\|_{L^{\infty}(E)}
\end{aligned}
$$




$$
\begin{aligned}
& =\left\|\left(\widetilde{I}_{h}-I\right) P_{K^{+}} \beta^{e}\right\|_{L^{\infty}\left(K^{+}\right)} \\
& \lesssim h^{2}\|\beta\|_{W_{\infty}^{2}\left({\widetilde{K^{+}}}^{l}\right)}
\end{aligned}
$$

Term III Since $\beta$ is a smooth tangent vector field on $\Gamma$ we have

$$
\left[n_{E} \cdot \beta^{e}\right]=\left[n_{E}\right] \cdot \beta^{e}=\left[n_{E}\right] \cdot t_{E}^{e}\left|t_{E}^{e} \cdot \beta^{e}\right|
$$

where $t_{E} \in T_{p(x)}(\Gamma)$ is the unit tangent vector at $p(x)$ to the exact surface that is orthogonal to edge $E$. Thus it remains to estimate $\left[n_{E}\right] \cdot t_{E}^{e}$. We have the identity

$$
\left[n_{E}\right] \cdot t_{E}^{e}=\left(\left[n_{K}\right] \times e_{E}\right) \cdot\left(n \times e_{E}\right)=\left[n_{K}\right] \cdot n \sim h^{2}
$$

where $e_{E}$ is the unit vector along $E$ directed in such a way that $n_{K^{+}} \times e_{E}=n_{E}^{+}$, $n_{K}=\left.n_{h}\right|_{K},\left[n_{K}\right]=n_{K^{+}}-n_{K^{-}}$, and we used the identity $n \cdot n_{h}=1+O\left(h^{2}\right)$.

(6.1-6.3). We obtain (6.1) by combining Remark 6.3 and (6.52). (6.2-6.3) follows in the same way.

\subsection{Error estimates}

Theorem 6.1 Let $u$ be the solution to (2.3) and $u_{h}$ the finite element approximation defined by (3.6), then the following estimate holds

$$
\|\| u^{e}-u_{h}\|\|_{h} \lesssim h^{3 / 2}\|u\|_{H^{2}(\Gamma)}+\left(C_{\beta}+C_{\alpha}\right) h^{2}\|u\|_{H^{1}(\Gamma)}+C_{f} h^{2}
$$

for all $h \in\left(0, h_{0}\right]$ with $h_{0}$ small enough.

Proof Adding and subtracting an interpolant

$$
\left\|\left|u^{e}-u_{h}\right|\right\|_{h} \leq\|\| u^{e}-\pi_{h} u^{e}\left\|_{h}+\right\| \mid \pi_{h} u^{e}-u_{h} \|_{h}
$$

Here the first term is estimated using the interpolation error estimate (4.28) and for the second we apply the Strang Lemma 6.1 together with the quadrature error estimates in Lemma 6.2.

Theorem 6.2 Let $u$ be the solution to (2.3) and $u_{h}$ the finite element approximation defined by (3.6), then the following estimate holds

$$
\left\|\nabla_{\Gamma_{h}}\left(u^{e}-u_{h}\right)\right\|_{\mathcal{K}_{h}} \lesssim h^{3 / 4}\|u\|_{H^{2}(\Gamma)}+\left(C_{\beta}+C_{\alpha}\right) h^{5 / 4}\|u\|_{H^{1}(\Gamma)}+C_{f} h^{5 / 4}
$$

for all $h \in\left(0, h_{0}\right]$ with $h_{0}$ small enough.

Proof We have the estimates

$$
\begin{aligned}
\left\|\nabla_{\Gamma_{h}}\left(u^{e}-u_{h}\right)\right\|_{\mathcal{K}_{h}} & \lesssim\left\|\nabla_{\Gamma_{h}}\left(u^{e}-\pi_{h} u^{e}\right)\right\|_{\mathcal{K}_{h}}+\left\|\nabla_{\Gamma_{h}}\left(\pi_{h} u^{e}-u_{h}\right)\right\|_{\mathcal{K}_{h}} \\
& \lesssim\left\|\nabla_{\Gamma_{h}}\left(u^{e}-\pi_{h} u^{e}\right)\right\|_{\mathcal{K}_{h}}+h^{-3 / 4}\left\|\mid \pi_{h} u^{e}-u_{h}\right\|_{h}
\end{aligned}
$$




$$
\begin{aligned}
& \lesssim\left\|\nabla_{\Gamma_{h}}\left(u^{e}-\pi_{h} u^{e}\right)\right\|_{\mathcal{K}_{h}}+h^{-3 / 4}\|\| \pi_{h} u^{e}-u^{e} \|_{h} \\
& \quad+h^{-3 / 4}\left\|\mid u^{e}-u_{h}\right\|_{h} \\
& \lesssim h\|u\|_{H^{2}(\Gamma)}+h^{-3 / 4} h^{3 / 2}\|u\|_{H^{2}(\Gamma)} \\
& \quad+h^{-3 / 4}\left(h^{3 / 2}\|u\|_{H^{2}(\Gamma)}+\left(C_{\beta}+C_{\alpha}\right) h^{2}\|u\|_{H^{1}(\Gamma)}+C_{f} h^{2}\right) \\
& \lesssim h^{3 / 4}\|u\|_{H^{2}(\Gamma)}+\left(C_{\beta}+C_{\alpha}\right) h^{5 / 4}\|u\|_{H^{1}(\Gamma)}+C_{f} h^{5 / 4}
\end{aligned}
$$

Here we added and subtracted an interpolant and used the triangle inequality, used the stability estimate in Proposition 5.2, added and subtracted an interpolant in the second term and used the triangle inequality, used interpolation error estimates (4.25) and (4.28) to estimate the first and the second term and finally Theorem 6.1 to estimate the third term, which conclude the proof of the desired estimate.

Remark 6.5 We note that these two error estimates are completely analogous to the estimates obtained in [3] for the corresponding method on standard triangular meshes in the plane.

\section{Condition number estimate}

Let $\left\{\varphi_{i}\right\}_{i=1}^{N}$ be the standard piecewise linear basis functions associated with the nodes in $\mathcal{T}_{h}$ and let $\mathcal{A}$ be the stiffness matrix with elements $a_{i j}=A_{h}\left(\varphi_{i}, \varphi_{j}\right)$. We recall that the condition number is defined by

$$
\kappa_{h}(\mathcal{A}):=|\mathcal{A}|_{\mathbb{R}^{N}}\left|\mathcal{A}^{-1}\right|_{\mathbb{R}^{N}}
$$

Using the ideas introduced in [6], we may prove the following bound on the condition number of the matrix.

Theorem 7.1 The condition number of the stiffness matrix $\mathcal{A}$ satisfies the estimate

$$
\kappa_{h}(\mathcal{A}) \lesssim h^{-2}
$$

for all $h \in\left(0, h_{0}\right]$ with $h_{0}$ small enough.

Proof First we note that if $v=\sum_{i=1}^{N} V_{i} \varphi_{i}$ and $\left\{\varphi_{i}\right\}_{i=1}^{N}$ is the usual nodal basis on $\mathcal{T}_{h}$ then the following well known estimates hold

$$
c h^{-d / 2}\|v\|_{\mathcal{T}_{h}} \leq|V|_{\mathbb{R}^{N}} \leq C h^{-d / 2}\|v\|_{\mathcal{T}_{h}}
$$

It follows from the definition (7.1) of the condition number that we need to estimate $|\mathcal{A}|_{\mathbb{R}^{N}}$ and $\left|\mathcal{A}^{-1}\right|_{\mathbb{R}^{N}}$.

Estimate of $|\mathcal{A}|_{\mathbb{R}^{N}}$ We have

$$
|\mathcal{A} V|_{\mathbb{R}^{N}}=\sup _{W \in \mathbb{R}^{N} \backslash 0} \frac{(W, \mathcal{A} V)_{\mathbb{R}^{N}}}{|W|_{\mathbb{R}^{N}}}
$$




$$
\begin{aligned}
& =\sup _{w \in V_{h} \backslash 0} \frac{A_{h}(v, w)}{|W|_{\mathbb{R}^{N}}} \\
& \lesssim h^{d-2}|V|_{\mathbb{R}^{N}}
\end{aligned}
$$

Here we used the following continuity of $A_{h}(\cdot, \cdot)$

$$
\begin{aligned}
A_{h}(v, w) & \lesssim\left\|\beta_{h} \cdot \nabla v\right\|_{\mathcal{K}_{h}}\|w\|_{\mathcal{K}_{h}}+h\|\| v\|\|_{\mathcal{F}_{h}}\left|\|w \mid\| \mathcal{F}_{h}\right. \\
& \lesssim\left(h\left\|\beta_{h} \cdot \nabla_{\Gamma_{h}} v\right\|_{\mathcal{K}_{h}}^{2}+h\|\| v \|\left.\right|_{\mathcal{F}_{h}} ^{2}\right)^{1 / 2}\left(h^{-1}\|w\|_{\mathcal{K}_{h}}^{2}+h \||| w||_{\mathcal{F}_{h}}^{2}\right)^{1 / 2} \\
& \lesssim h^{d-2}|V|_{\mathbb{R}^{N}}|W|_{\mathbb{R}^{N}}
\end{aligned}
$$

In the last step we used the estimates

$$
\begin{aligned}
h\left\|\beta_{h} \cdot \nabla_{\Gamma_{h}} v\right\|_{\mathcal{K}_{h}}^{2}+h\|\| v \|\left.\right|_{\mathcal{F}_{h}} ^{2} & \lesssim\left\|\beta_{h} \cdot \nabla_{\Gamma_{h}} v\right\|_{\mathcal{T}_{h}}^{2}+\|\nabla v\|_{\mathcal{T}_{h}}^{2} \\
& \lesssim h^{-2}\|v\|_{\mathcal{T}_{h}}^{2} \lesssim h^{d-2}|V|_{\mathbb{R}^{N}}^{2}
\end{aligned}
$$

where we used the inverse estimates (4.9) and (4.8) to pass from $\mathcal{K}_{h}$ and $\mathcal{F}_{h}$ to $\mathcal{T}_{h}$, an inverse estimate to remove the gradient, and finally the equivalence (7.3); and

$$
h^{-1}\|w\|_{\mathcal{K}_{h}}^{2}+h\|\| w\left\|_{\mathcal{F}_{h}}^{2} \lesssim h^{-2}\right\| w\left\|_{\mathcal{T}_{h}}^{2}+\right\| \nabla w\left\|_{\mathcal{T}_{h}}^{2} \lesssim h^{-2}\right\| w \|_{\mathcal{T}_{h}}^{2} \lesssim h^{d-2}|W|_{\mathbb{R}^{N}}^{2}
$$

where we used the same sequence of estimates. It follows that

$$
|\mathcal{A}|_{\mathbb{R}^{N}} \lesssim h^{d-2}
$$

Estimate of $\left|\mathcal{A}^{-1}\right|_{\mathbb{R}^{N}}$ We note that using (7.3) and Lemma 5.1 we have

$$
h^{d}|V|_{\mathbb{R}^{N}}^{2} \lesssim\|v\|_{\mathcal{T}_{h}}^{2} \lesssim h\left(\|v\|_{\mathcal{K}_{h}}^{2}+\|\| v \|_{\mathcal{F}_{h}}^{2}\right) \lesssim\|\| v \|_{h}^{2}
$$

where in the last step we used the fact that $h \in\left(0, h_{0}\right]$ and the definition (4.4) of $\||\cdot|\|_{h}$. Starting from (7.13) and using the inf-sup condition (5.84) we obtain

$$
\begin{aligned}
\left.|V|_{\mathbb{R}^{N}} \lesssim h^{-d / 2}|\| v|\right|_{h} & \lesssim h^{-d / 2} \sup _{w \in V_{h} \backslash\{0\}} \frac{A_{h}(v, w)}{\left.|||w|\right|_{h}} \\
& \lesssim \sup _{W \in \mathbb{R}^{N} \backslash\{0\}} h^{-d / 2} \frac{|\mathcal{A} V|_{\mathbb{R}^{N}|W|_{\mathbb{R}^{N}}}}{h^{d / 2}|W|_{\mathbb{R}^{N}}} \lesssim h^{-d}|\mathcal{A} V|_{\mathbb{R}^{N}}
\end{aligned}
$$

Here we used (7.13), $h^{d / 2}|W|_{\mathbb{R}^{N}} \lesssim|||w|||_{h}$, to replace ||$|w|||_{h}$ by $h^{d / 2}|W|_{\mathbb{R}^{N}}$ in the denominator. Setting $V=\mathcal{A}^{-1} X, X \in \mathbb{R}^{N}$, we obtain

$$
\left|\mathcal{A}^{-1}\right|_{\mathbb{R}^{N}} \lesssim h^{-d}
$$

Conclusion The claim follows by using the bounds (7.12) and (7.16) in the definition (7.1). 


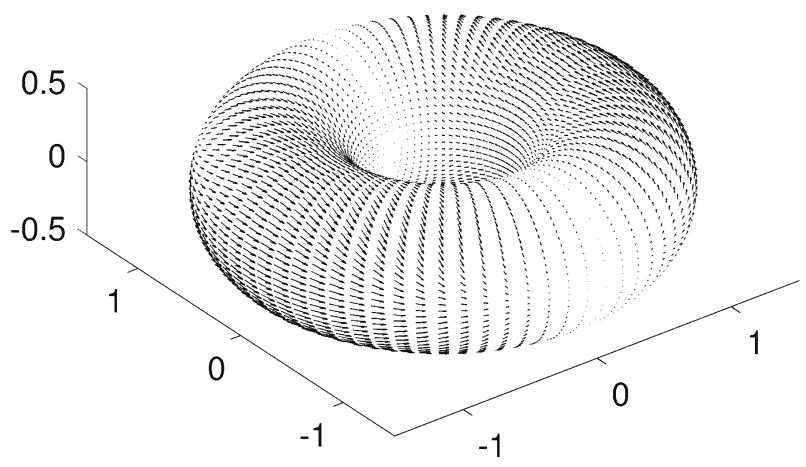

Fig. 1 The vector field $\beta$ on the torus

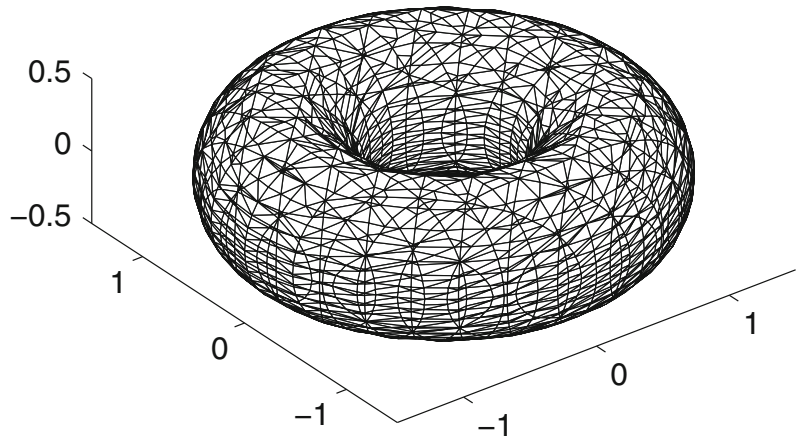

Fig. 2 The triangulation of $\Gamma_{h}$ for $h=0.2$

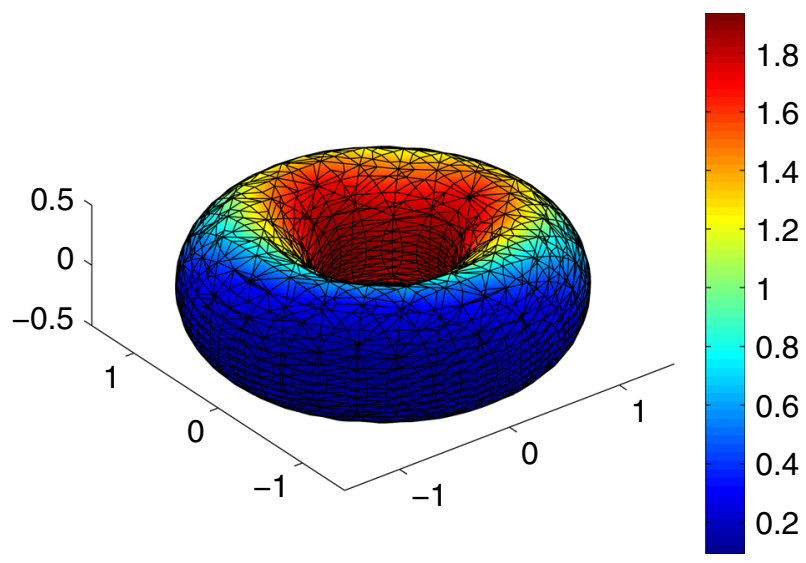

Fig. 3 The solution $u_{h}$ for $h=0.2$

\section{Numerical results}

We consider an example where the surface $\Gamma$ is a torus given by the zero level set of the the signed distance function $\rho=\left(z^{2}+\left(\left(x^{2}+y^{2}\right)^{1 / 2}-R\right)^{2}\right)^{1 / 2}-r$, with $R=1$ 


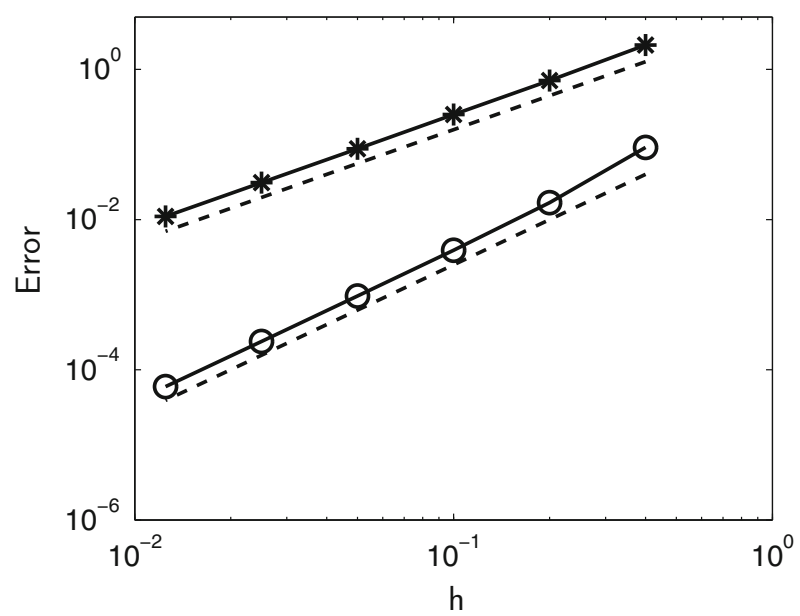

Fig. 4 Convergence of $u_{h}$. Circles represent the error measured in the $L^{2}$ norm $\left(\left\|u^{e}-u_{h}\right\|_{\mathcal{K}_{h}}\right)$ and stars represent the error in the energy norm $\left(\left\|\left|u^{e}-u_{h}\right|\right\|_{h}\right)$. The dashed lines are proportional to $h^{2}$ and $h^{3 / 2}$

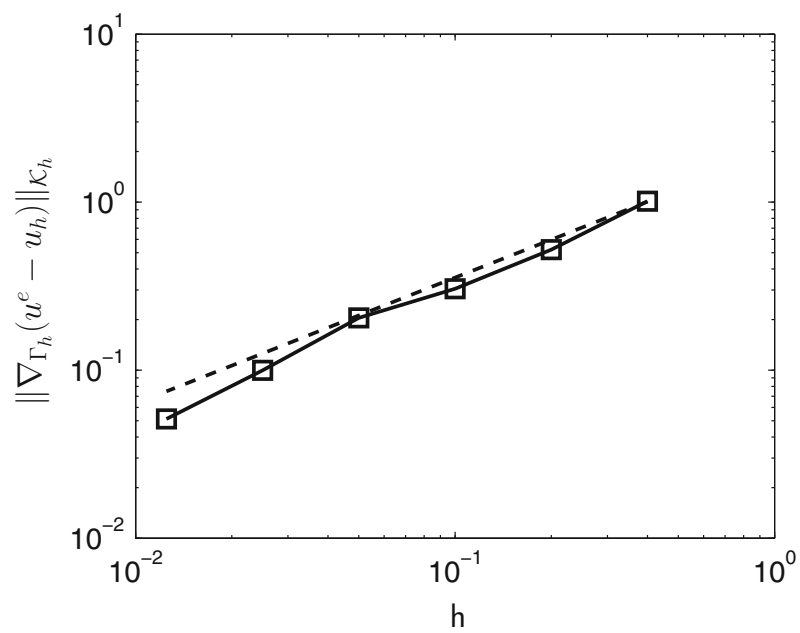

Fig. 5 Convergence of $\left\|\nabla_{\Gamma_{h}}\left(u^{e}-u_{h}\right)\right\|_{\mathcal{K}_{h}}$. The dashed line is proportional to $h^{3 / 4}$

and $r=1 / 2$. We choose $\alpha=1$,

$$
\beta=P_{\Gamma}\left(x^{2} y z, x, y z^{3}\right)
$$

and $f$ such that the exact solution is

$$
u=\left(0.5 x+(x-1)^{2}+0.5 y+(y-1)\right) e^{(-x(x-1)-y(y-1))}
$$




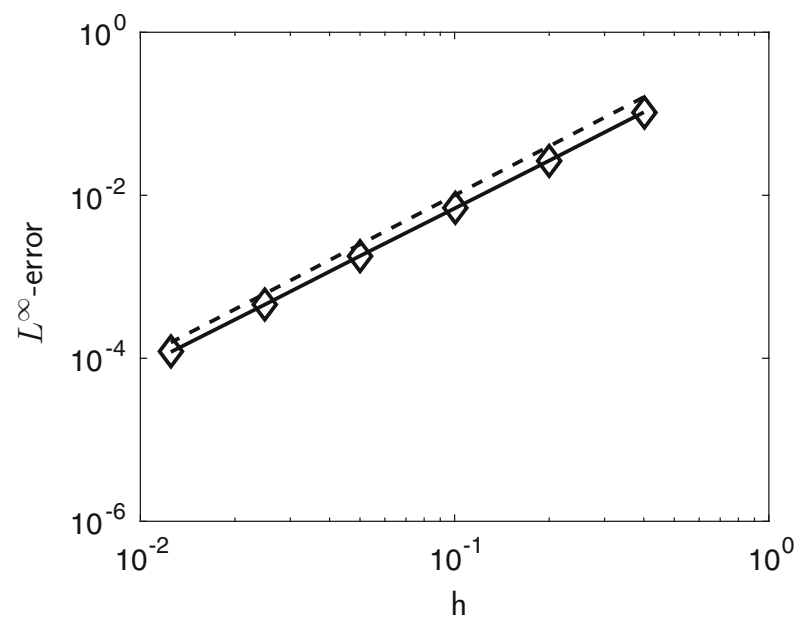

Fig. 6 Convergence of $\left\|u^{e}-u_{h}\right\|_{L^{\infty}\left(\mathcal{K}_{h}\right)}$. The dashed line is $y=h^{2}$

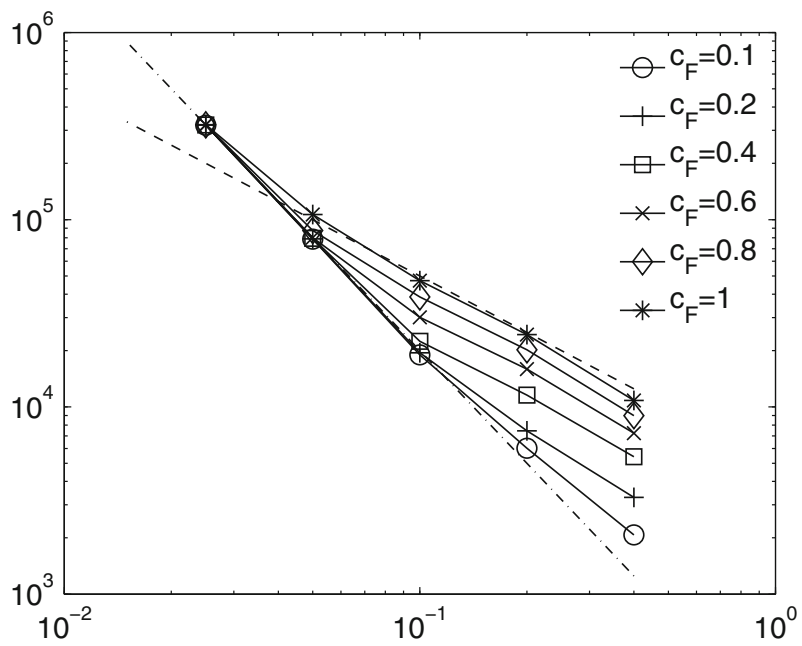

Fig. 7 The condition number of the matrix versus mesh size. The dashed lines are proportional to $h^{-1}$ and $h^{-2}$

The vector field $\beta$ is shown in Fig. 1. A structured mesh $\mathcal{T}_{0, h}$ consisting of tetrahedra on the domain $[-1.6,1.6] \times[-1.6,1.6] \times[-0.6,0.6]$ is generated independently of the position of the torus. The mesh size parameter is defined as $h=h_{x}=h_{y}=h_{z}$. An approximate distance function $\rho_{h}$ is constructed using the nodal interpolant $\pi_{h} \rho$ on the background mesh and $\Gamma_{h}$ is the zero levelset of $\rho_{h}$ and $n_{h}$ is the piecewise constant unit normal to $\Gamma_{h}$. The triangulation of $\Gamma_{h}$ is shown in Fig. 2. We use the proposed method with the stabilization parameter chosen as $c_{F}=10^{-2}$. A direct solver was used to solve the linear systems. The solution $u_{h}$ for $h=0.2$ is shown in Fig. 3 . 


\subsection{Convergence study}

We compare our approximation $u_{h}$ with the exact solution $u$ given in Eq. (8.2). The convergence of $u_{h}$ in the $L^{2}$-norm and the energy norm are shown in Fig. 4. We observe second order convergence in the $L^{2}$-norm and as expected a convergence order of 1.5 in the energy norm. The error in the gradient $\left\|\nabla_{\Gamma_{h}}\left(u^{e}-u_{h}\right)\right\|_{\mathcal{K}_{h}}$ versus mesh size is shown in Fig. 5 and the observed convergence order is slightly better than $3 / 4$ for the finest meshes. Our analysis does not cover the $L^{\infty}$-convergence but in Fig. 6 we show the $L^{\infty}$-errors versus mesh size and observe around second order convergence.

\subsection{Condition number study}

We compute the condition number of the stiffness matrix for a sequence of uniformly refined meshes and different values of the stabilization parameter $c_{F}$. We find that the asymptotic behavior as the meshsize tend to zero is $O\left(h^{-2}\right)$ as expected from the analysis, while on coarser meshes for larger values of $c_{F}$ the behavior is closer to $O\left(h^{-1}\right)$, see Fig. 7.

Open Access This article is distributed under the terms of the Creative Commons Attribution 4.0 International License (http://creativecommons.org/licenses/by/4.0/), which permits unrestricted use, distribution, and reproduction in any medium, provided you give appropriate credit to the original author(s) and the source, provide a link to the Creative Commons license, and indicate if changes were made.

\section{References}

1. Burman, E., Ern, A., Fernández, M.A.: Explicit Runge-Kutta schemes and finite elements with symmetric stabilization for first-order linear PDE systems. SIAM J. Numer. Anal. 48(6), 2019-2042 (2010)

2. Burman, E., Fernández, M.A.: Finite element methods with symmetric stabilization for the transient convection-diffusion-reaction equation. Comput. Methods Appl. Mech. Eng. 198(33-36), 2508-2519 (2009)

3. Burman, E., Hansbo, P.: Edge stabilization for Galerkin approximations of convection-diffusionreaction problems. Comput. Methods Appl. Mech. Eng. 193(15-16), 1437-1453 (2004)

4. Burman, E., Hansbo, P.: Fictitious domain finite element methods using cut elements: I. A stabilized Lagrange multiplier method. Comput. Methods Appl. Mech. Eng. 199(41-44), 2680-2686 (2010)

5. Burman, E., Hansbo, P.: Fictitious domain finite element methods using cut elements: II. A stabilized Nitsche method. Appl. Numer. Math. 62(4), 328-341 (2012)

6. Burman, E., Hansbo, P., Larson, M.G.: A stabilized cut finite element method for partial differential equations on surfaces: the Laplace-Beltrami operator. Comput. Methods Appl. Mech. Eng. 285, 188207 (2015)

7. Burman, E., Hansbo, P., Larson, M.G., Massing, A.: A cut discontinuous Galerkin method for the Laplace-Beltrami operator. IMA J. Numer. Anal. 37(1), 138-169 (2017). https://doi.org/10.1093/ imanum/drv068

8. Burman, E., Hansbo, P., Larson, M.G., Zahedi, S.: Cut finite element methods for coupled bulk-surface problems. Numer. Math. 133(2), 203-231 (2016)

9. Chernyshenko, A.Y., Olshanskii, M.A.: An adaptive octree finite element method for PDEs posed on surfaces. Comput. Methods Appl. Mech. Eng. 291, 146-172 (2015)

10. Deckelnick, K., Dziuk, G., Elliott, C.M., Heine, C.-J.: An $h$-narrow band finite-element method for elliptic equations on implicit surfaces. IMA J. Numer. Anal. 30(2), 351-376 (2010)

11. Demlow, A.: Higher-order finite element methods and pointwise error estimates for elliptic problems on surfaces. SIAM J. Numer. Anal. 47(2), 805-827 (2009) 
12. Demlow, A., Olshanskii, M.A.: An adaptive surface finite element method based on volume meshes. SIAM J. Numer. Anal. 50(3), 1624-1647 (2012)

13. Dziuk, G.: Finite elements for the Beltrami operator on arbitrary surfaces. In: Hildebrandt, S., Leis, R. (eds.) Partial Differential Equations and Calculus of Variations, volume 1357 of Lecture Notes in Mathematics, pp. 142-155. Springer, Berlin (1988)

14. Dziuk, G., Elliott, C.M.: Eulerian finite element method for parabolic PDEs on implicit surfaces. Interfaces Free Bound. 10(1), 119-138 (2008)

15. Dziuk, G., Elliott, C.M.: Finite element methods for surface PDEs. Acta Numer. 22, 289-396 (2013)

16. Hansbo, A., Hansbo, P., Larson, M.G.: A finite element method on composite grids based on Nitsche's method. M2AN Math. Model. Numer. Anal. 37(3), 495-514 (2003)

17. Hansbo, P., Larson, M.G., Larsson, K.: Analysis of finite element methods for vector Laplacians on surfaces. Technical report, Mathematics, Umeå University, Sweden (2016). arXiv:1610.06747

18. Hansbo, P., Larson, M.G., Zahedi, S.: A cut finite element method for a Stokes interface problem. Appl. Numer. Math. 85, 90-114 (2014)

19. Hansbo, P., Larson, M.G., Zahedi, S.: Characteristic cut finite element methods for convection-diffusion problems on time dependent surfaces. Comput. Methods Appl. Mech. Eng. 293, 431-461 (2015)

20. Hansbo, P., Larson, M.G., Zahedi, S.: A cut finite element method for coupled bulk-surface problems on time-dependent domains. Comput. Methods Appl. Mech. Eng. 307, 96-116 (2016)

21. Massing, A., Larson, M.G., Logg, A., Rognes, M.E.: A stabilized Nitsche fictitious domain method for the Stokes problem. J. Sci. Comput. 61(3), 604-628 (2014)

22. Olshanskii, M.A., Reusken, A.: Error analysis of a space-time finite element method for solving PDEs on evolving surfaces. SIAM J. Numer. Anal. 52(4), 2092-2120 (2014)

23. Olshanskii, M.A., Reusken, A., Grande, J.: A finite element method for elliptic equations on surfaces. SIAM J. Numer. Anal. 47(5), 3339-3358 (2009)

24. Olshanskii, M.A., Reusken, A., Xu, X.: An Eulerian space-time finite element method for diffusion problems on evolving surfaces. SIAM J. Numer. Anal. 52(3), 1354-1377 (2014)

25. Olshanskii, M.A., Reusken, A., Xu, X.: A stabilized finite element method for advection-diffusion equations on surfaces. IMA J. Numer. Anal. 34(2), 732-758 (2014) 